\title{
ESTRUCTURA Y COMPOSICIÓN FLORÍSTICA DEL BOSQUE DE LA LLANURA ALUVIAL INUNDABLE DE LA AMAZONÍA PERUANA: II. EL SOTOBOSQUE DE LA RESTINGA
}

\author{
Gustav Nebel ${ }^{1}$, Jens Dragsted ${ }^{1}$ y Jerome K. Vanclay ${ }^{2}$
}

\begin{abstract}
RESUMEN
En este documento se describen la estructura y la composición florística de pequeños árboles y arbustos (1,5 m de altura y hasta $10 \mathrm{~cm}$ de DAP), de dos bosques de la llanura aluvial inundable del bajo Ucayali, en la Amazonía Peruana. Estos bosques son del tipo restinga alta y restinga baja, con una inundación anual promedio de alrededor de 1 a 2 meses, respectivamente. Los suelos son entisoles ricos en nutrientes, y la vegetación arbórea forma altas copas cerradas con la presencia de árboles emergentes. Se establecieron un total de 25 parcelas permanentes de muestreo cubriendo un área de 0,64 ha. Estas fueron colocadas dentro de seis parcelas permanentes de muestreo de una hectárea cuadrada, en donde se inventariaron individuos $(>10 \mathrm{~cm}$ DAP). La densidad promedio total y el área basal del sotobosque es de 4458 plantas/ ha y $5,0 \mathrm{~m}^{2} /$ ha, respectivamente. Dentro de las familias de árboles más importantes están Moraceae, Leguminosae, Annonaceae, Euphorbiaceae y Lauraceae; mientras que las familias de árboles pequeños y arbustos importantes son Violaceae, Rubiaceae, Melastomataceae y Olacaceae. De un total de 264 especies arbóreas, 208 se registraron en la restinga de estrato superior y 204 se registraron en la restinga de sotobosque. El 56\% de las especies se encuentran compartiendo los dos estratos de bosque, mientras que cerca del $22 \%$ están confinados a uno de ellos solamente. Las especies presentes solamente en el sotobosque son predominantemente arbustos o arbolitos, mientras que algunas de las especies, presentes solamente en el estrato superior, son probablemente especies de sucesión temprana casi por desaparecer de los bosques.
\end{abstract}

Palabras claves: Zonas húmedas, valor de importancia por familia, valor de importancia por especie, biodiversidad, riqueza de especies, uniformidad de especies.

1 Real Universidad de Veterinaria y Agricultura. Departamento de Economía y Recursos Naturales. Unidad de Forestales. Rolighedsvej 23, 1958 Frederiksberg C, Dinamarca.

2 Centro Internacional para la Investigación Forestal, P.O. Box 6596, JKPWB, Jakarta 10065, Indonesia. 


\begin{abstract}
Structure and floristic composition of small trees and shrubs $(1,5 \mathrm{~m}$ height to $0 \mathrm{~cm}$ $\mathrm{DBH})$ are described in two flood plain forests of the lower Ucayali river, Peruvian Amazon. The forests are of high and low restinga type, on an annual average flooded around 1 and 2 months, respectively. The soils are nutrients rich entisolls, and the vegetation forms closed highcanopy forests with presence of emergents. A total of 25 permanent sample plots covering 0,64 ha were established. They are nested within six quadratic one- hectare permanent sample plots where large individuals $(>=10$ $\mathrm{cm} \mathrm{DBH)} \mathrm{were} \mathrm{inventoried.} \mathrm{Overall} \mathrm{average} \mathrm{density} \mathrm{and} \mathrm{basal} \mathrm{area} \mathrm{of} \mathrm{the} \mathrm{understory}$ is 4 458/ha and 5,0 $\mathrm{m}^{2} /$ ha, respectively. The families of Moraceae, Leguminosae, Annonaceae, Euphorbiaceae, and Lauraceae are among the most important tree families, while important shrub and small tree families are Violaceae, Rubiaceae, Melastomataceae, and Olacaceae. Doscientos ocho and 204 tree species out of a total of 264 are registered in the restinga forest over and understories, respectively. Cincuentiseis por ciento of the species are shared between the two forest strata, while around $22 \%$ are confined to each of them. Species present only in the understorey are predominantly shrubs of treelets, while some of the species with a presence only in overstorey are probably early succession species about to disappear from the forests.
\end{abstract}

\title{
1. INTRODUCCIÓN
}

Dentro de la composición de un bosque, al sotobosque justifica ponerle especial atención, debido a que a menudo contiene más especies vegetales y contribuye más a la cadena de alimentos que otros estratos. Las especies pueden estar restringidas a este estrato de bosque, y estos pequeños individuos pueden proveer refugio y comida a muchos animales (Foster, 1982; Gentry y Emmons, 1986; Hubbel y Foster, 1992). La regeneración de los bosques tiene lugar a través de la presencia de plántulas y plantones en los sotobosques, claros o en campo abierto, según sea la especie (Denslow, 1980; Whitmore et al., 1983; Swaine y Whitmore, 1989; Clark y Clark, 1992). Además, dentro de sus nichos, los pequeños individuos pueden ser importantes en procesos tales como el reciclaje de nutrientes (Jordan, 1985). Esto implica que es necesario conocer la estructura, la composición florística y la dinámica de los pequeños individuos con el fin de desarrollar sistemas silviculturales (Lamprecht, 1989; GómezPompa y Burley, 1991; Hubbell y Foster, 1992; Whitmore, 1995).

La mayoría de los inventarios botánicos cuantitativos realizados en la Amazonía peruana se han concentrado en individuos mayores en lugar de pequeños individuos 
(Ulh y Murphy, 1981; Boom, 1986; Campbell et al., 1986; Blaslev et al., 1987; Rankin-de-Mérona, 1992; Ayres, 1995; Valencia et al., 1994). Sin embargo, algunos trabajos han incluido pequeños individuos en sus inventarios (Worbes, 1983, 1986; Gentry y Emmons, 1987; Colonello, 1990; Worbes et al., 1992; Freitas, 1996a, 1996b). Muchos de estos estudios tienen que ver con bosques no inundados, mientras que los estudios de Worbes (1983, 1986), Gentry y Emmons (1987), Colonello (1990), Worbes et al. (1992) y Freitas (1996a) se relacionan con los bosques de llanos inundables amazónicos.

El presente estudio proporciona una descripción cuantitativa de la estructura y composición florística de individuos arbóreos de menor tamaño y de arbustos en dos bosques de llanos inundables amazónicos, en el bajo Ucayali, Perú. Se hicieron comparaciones, en el mismo sitio, con los árboles de estratos más altos (árboles con DAP $>10 \mathrm{~cm}$ ). Además, se clasificaron las especies con suficiente densidad de acuerdo a su tamaño máximo alcanzado. Los términos pequeños individuos o sotobosque se usan para identificar individuos con tamaños en el rango de $1,5 \mathrm{~m}$ de altura hasta 10 $\mathrm{cm}$ de DAP, mientras que los términos individuos mayores o de estratos altos se refieren a individuos con más de $10 \mathrm{~cm}$ de DAP.

\section{EL ÁREA DE ESTUDIO}

El estudio se realizó en el departamento de Loreto - Perú, localizado en las zonas bajas húmedas tropicales del Amazonas. Las parcelas se establecieron en los bosques de llanos inundables de restinga alta y restinga baja, en la zona de BragaSupay del Bajo Ucayali, aproximadamente a $10 \mathrm{~km}$ al sur oeste de Jenaro Herrera $\left(4^{\circ} 55^{\prime} \mathrm{S}, 73^{\circ} 44^{\prime} \mathrm{O}\right)$. Los aspectos generales del sitio de estudio son descritos por Kvist y Nebel (en este documento), mientras que el lugar, las condiciones de crecimiento y la composición florística y estructura del bosque de estrato superior, en los dos bosques de restinga, son descritos por Nebel et al. (en este documento).

Ambos bosques de restinga se caracterizan por ser de suelos entisoles relativamente fértiles, clasificados como Typic Hidraquents (Andersen, 1995). Durante septiembre a febrero de 1997, la inundación promedio fue alrededor de uno a dos meses por año, para restinga alta y baja, respectivamente. Ambos tipos pueden soportar bosques altos, con una altura de dosel de aproximadamente $30 \mathrm{~m}$, además de algunos pocos y separados árboles emergentes por encima del dosel principal. Aparentemente los bosques no han sido intervenidos por el hombre, pero los individuos de alto valor comercial parecen haber sido talados. 


\section{MATERIALES Y MÉTODOS}

Con el fin de estudiar los árboles pequeños y los arbustos en los bosques de restinga, se establecieron las parcelas permanentes de muestreo durante setiembre a noviembre de 1993. Se establecieron un total de 25 sub-parcelas de 16x16 m, dentro de seis parcelas permanentes de muestreo de una hectárea cuadrada. Cuatro sub-parcelas de $16 \times 16$ m fueron establecidas en cada una de las seis parcelas de una hectárea, y una sub-parcela de 16×16 m adicional fue establecida en la parcela 1 (restinga alta), con el fin de muestrear 1024 y $1280 \mathrm{~m}^{2}$, respectivamente. Las sub-parcelas fueron sistemáticamente distribuidas a lo largo y ancho de las parcelas de una hectárea.

Los árboles y arbustos de $1,5 \mathrm{~m}$ de altura y $10 \mathrm{~cm}$ de DAP fueron permanentemente marcados con etiquetas de aluminio numeradas, registrándose sus DAP y alturas totales. Para medir los diámetros de los individuos con DAP menores a $5 \mathrm{~cm}$, se utilizó un calibrador Vernier, y en el caso de los individuos de 5 a $10 \mathrm{~cm}$ DAP se utilizó una cinta diamétrica. Los individuos con una altura total de hasta $15 \mathrm{~m}$ se midieron con una vara telescópica, y aquellos más altos fueron visualmente estimados. La posición y la forma de la copa fueron evaluadas de acuerdo a la clasificación de Dawkins (Alder y Synnott, 1992).

Todos los individuos presentes en las sub-parcelas fueron identificados en el campo. En el caso de que la clasificación de campo no hubiese sido propiamente realizada, se colectaron muestras de especímenes, tal fue el caso para el $63 \%$ de los individuos. La identificación de estos especímenes se llevó a cabo en el Herbario de la Universidad de Aarhus (AAU) en Dinamarca, y muchos de los especímenes a nivel de familia y género fueron enviados a especialistas taxónomos para su identificación. En el caso de no contar con la muestra del espécimen o de que ésta se hubiese perdido, o que el individuo se hubiese muerto durante el periodo de establecimiento de la parcela hasta su colección, o de que hubiese sido imposible su identificación, los individuos se registraron como desconocidos.

Los cálculos del índice de valor de importancia de la familia (FIV) se hicieron según Mori et al. (1983), mientras que el índice de valor de importancia de la especie (SIV) se calculó de acuerdo a Curtis y McIntosh $(1950,1951)$. La frecuencia relativa se estimó utilizando unidades de muestreo de $8 \times 8 \mathrm{~m}$. Se asume que los especímenes desconocidos ya están representados, por lo tanto, no se les considera en el cálculo de las frecuencias. Los coeficientes de similitud de Jaccard y Sorensen se calcularon según Greig-Smith (1983) y Sorensen (1948). Las fórmulas para el FIV, SIV y los coeficientes de similitud están dados por Nebel et al. (en este documento). 


\section{RESULTADOS}

La densidad de los individuos del sotobosque está alrededor de 4400 plantas/ha en ambos bosques, y el área basal cerca de $5 \mathrm{~m}^{2} /$ ha (Cuadro 1). En las Figuras 1 y 2 se muestra la distribución del tamaño de los individuos.

En las 0,64 ha cubiertas por el estudio, se encuentran presentes un total de 47 familias, mientras que 35-43 familias están representadas en cada una de las parcelas de una hectárea. De un total de 204 especies registradas, 87-109 se encuentran en parcelas simples de una hectárea en donde se establecieron cuatro sub-parcelas de 16×16 $\mathrm{m}$, mientras que 120 están presentes en parcela de una hectárea con cinco sub-parcelas de 16x16 m (Cuadro 1). Aproximadamente el 10\% de las especies comprenden cerca del 50\% de los individuos (Figura 3). Un patrón similar se observa cuando se usa el área basal en lugar de la densidad.

Muchas de estas especies pueden ser encontradas en más de una parcela, ya sea dentro o entre los dos tipos diferentes de bosque (Cuadro 2).

El 19\% de las especies fueron encontradas en restinga alta solamente, mientras que el $21 \%$ son exclusivos de la restinga baja. Estos dos tipos de bosque tienen altos coeficientes de similitud (Cuadro 3).

El número de especies se incrementa rápidamente cuando el tamaño de la muestra se incrementa en aproximadamente 0,1 ha, para tamaños mayores se observa un incremento gradual pero constante de nuevas especies por unidad de área.

Las densidades relativas, diversidades y dominancias, así como los FIV resultantes para los bosques de restinga alta y baja se muestran en el Cuadro 4. Los SIV y sus componentes se adjuntan en el Apéndice 1.

Una gran cantidad de las especies arbóreas del sotobosque también están presentes en los estratos superiores (Cuadro 5). De las 264 especies arbóreas presentes en el sotobosque y los estratos superiores de los bosques de restinga, $148(56 \%)$ están compartidos entre los dos tipos de estrato forestal, mientras que 56 (21\%) y 60 (23\%) especies están restringidas a individuos pequeños y mayores, respectivamente. En los bosques de restinga 226 especies ( $86 \%$ ) son comparativamente pequeños y no se han observado DAP que excedan el máximo de $50 \mathrm{~cm}$ (Figura 5).

El Apéndice 2 clasifica las especies arbóreas del bosque de restinga (representado por 10 o más individuos) de acuerdo al máximo diámetro alcanzado (MaxDAP). 
Las clases diamétricas utilizadas se basan en la clasificación utilizada por otros investigadores (Campbell et al., 1986; Swaine et al., 1987; Hubbell y Foster, 1992; Richards, 1996).

\section{DISCUSIÓN}

Brunig (1983), encontró una considerable variación en la densidad del bosque húmedo tropical (2 000-20 000/ha) en el rango del DAP de alrededor de $1-10 \mathrm{~cm}$, aunque el promedio se sitúa cerca del menor valor. Para este mismo rango de diámetro, Bongers et al. (1988) reportó densidades comparables (2 250-5 000/ha) en las zonas bajas de los bosques húmedos tropicales. Gentry y Terbourg (1990) registraron 203 individuos (2,5-10 cm DAP) en una parcela de 0,1 ha en Cocha Cashu, en la selva baja inundable peruana, y mencionan que el bosque tenía un sotobosque abierto debido, generalmente, a la falta de individuos en la clase diamétrica de 2,5$10 \mathrm{~cm}$ de DAP. Comparando los resultados de los bosques de restinga de BragaSupay (Cuadro 1, Figura 1) con las cifras mencionadas líneas arriba encontramos que ellos están dentro del rango común para las zonas bajas de los bosques húmedos tropicales, y los datos no sugieren una restricción en el desarrollo del sotobosque, tal como se podría pensar que sería una consecuencia de la inundación anual. Las inundaciones más largas ocurren en el bosque de restinga baja, el cual ostenta la mayor proporción de individuos en la menor clase de altura (Figura 2). Sin embargo, parece que los bosques adyacentes, expuestos a inundaciones más largas tienen menos individuos de sotobosque.

Ocho de las diez familias más importantes están compartidas. En la restinga alta las familias Violaceae y Arecaceae están en el segundo y décimo puesto de importancia, mientras que en la restinga baja se posicionan en el número veintiuno y diecisiete, respectivamente. Por otra parte, la familia Moraceae es mucho más importante en la restinga alta que en la baja. En la restinga baja las familias Euphorbiaceae, Myrtaceae, Olacaceae y Flacourtiaceae alcanzan valores considerablemente más altos que en la restinga alta. Del total de las familias de especies arbóreas, la Moraceae, Leguminosae, Annonaceae, Euphorbiaceae, y Lauraceae están dentro de las más importantes. También están dentro de las más importantes las familias Violaceae, Rubiaceae, Melastomataceae y Olacaceae que contienen especies de arbustos y de árboles más pequeños.

En ambos bosques de restinga son importantes las especies de mediana altura como Oxandra sphaerocarpa, Drypetes amazonica y Perebea longipedunculata. En am- 
bos bosques también se pueden encontrar varios arbustos de importancia como Tococa sp., Neea floribunda, Heisteria acuminata y Coussarea brevicaulus. Algunas especies pueden alcanzar mayores dimensiones, especialmente la palma Scheelea brachyclada, así como Guatteria sp. 1, Protium nodulosum, Inga cinnamomea, Maquira coreacea. Sorocea steinbachii y Leonia glycicarpa, son representativas de la restinga alta. Igualmente, entre las especies de menor altura, Naucleopsis glabra, Rondeletia sp. y Gloeospermum equatoriense son indicadores de las parcelas de restinga alta. En la restinga baja son muy importantes: Zygia juruana, de crecimiento mayor, y Laetia corymbulosa, Myrcia sp. 5 y Rudgea sessiliflora, especies de menor altura. Algunas de estas especies son distintas y pueden ser útiles para distinguir entre los tipos de bosques (Apéndice 1).

Gentry y Emmons (1987) estudiaron los sotobosques de los bosques pluviales neotropicales, incluyendo la regularmente inundada Cocha Cashu de la selva baja inundable peruana. Ellos encontraron que muchos arbustos tienden a estar presentes en lugares fértiles más que en sitios infértiles, en donde los plantones de los árboles dominan el sotobosque. Concordantemente, observamos muchos arbustos y especies de mediana altura (Cuadro 5, Apéndice 2) en los bosques fértiles de la restinga de Braga-Supay. En los bosques altos adyacentes más cercanos, que están expuestos a inundaciones más prolongadas, muy pocas especies de arbustos tienden a estar presentes.

Muchas especies presentes en los bosques de Braga-Supay solamente tienen diámetros menores a $10 \mathrm{~cm}$ DAP (Figura 5, Cuadro 5), lo cual concuerda con los hallazgos de Gentry y Dodson (1987). Asimismo, se tiene la presencia de varias especies con individuos que tienen el DAP por encima de los $10 \mathrm{~cm}$ solamente (Cuadro 5). Sin embargo, en estas proporciones pueden haber especies que accidentalmente vienen a estar representados por individuos ya sea pequeños o desarrollados solamente. En realidad, al considerar especies presentes con más de 10 individuos, la presencia de 15 de ellos está restringida al sotobosque, mientras que 9 están solamente registrados en el estrato superior (cf. 60 y 56 cuando se consideran todas las especies Cuadro 5, Apéndice 2). Una especie puede estar presente (1) en el estrato superior y en sotobosque, (2) en el sotobosque, o (3) en el estrato superior. Bajo las condiciones ambientales representadas por la muestra y asumiendo que las observaciones no se han hecho al azar solamente, (1) indica que la especie crece hasta tamaños mayores y regenera; (2) sugiere que es un arbusto/un arbolito, o un invasor luchando bajo las actuales condiciones ambientales, p.ej. debido al desarrollo de la sucesión; (3) simboliza que las especies regeneran bajo diferentes condiciones ambientales, p.ej. en una etapa previa de sucesión. Las especies en la restinga de Braga-Supay que perte- 
necen a (2) son principalmente arbustos o arbolitos. En contraste, muchas especies de (3) son árboles de gran crecimiento sin individuos del tamaño de plantones o postes, los cuales pueden desaparecer de los bosques. Anaxagorea sp., Apeiba aspera, Calycophyllum spruceanum, Ceiba pentandra, Cordia lutea, Inga edulis y Pseudobombax munguuba son algunas de las especies que pertenecen a este grupo. Se sabe que la mayoría de estas especies están confinadas a las tempranas etapas de sucesión. Esto indica que los bosques de restinga de Braga-Supay están en la etapa de desarrollo de la sucesión, el cual probablemente está más avanzado en la restinga alta, donde los grandes árboles de Calycophyllum spruceanum ya no están representados. Esta observación está en buena concordancia con Foster et al. (1986), Salo et al. (1986), Worbes et al. (1992) y Worbes (1997), quienes observaron que la composición florística y estructura de los bosques de selva baja están fuertemente influenciados por la etapa de sucesión. Mayor evidencia de esta observación también la da Nebel et al. (en este documento), quien afirma que la restinga alta es una etapa de sucesión posterior a la restinga baja.

Si se contara con muestras más grandes se podría obtener, a partir de estudios detallados de sus patrones de distribución de diámetros, una nueva impresión de cómo luchan las especies solitarias (Denslow, 1980; Whitmore et al., 1983; Bongers et al., 1988; Swaine y Whitmore, 1989; Nebel et al., en este documento).

\section{CONCLUSIONES}

En los bosques húmedos tropicales se está incrementado el establecimiento de parcelas permanentes de muestreo con el fin de investigar la composición florística, la estructura y los procesos de la dinámica del bosque. Hemos encontrado que, utilizando el sub-muestreo dentro de las parcelas en donde existen individuos de estrato superior bajo estudio, es posible describir con éxito los patrones de la composición florística y estructura del sotobosque y su relación con los datos registrados para el estrato superior. Esto ayuda a completar la descripción de la biodiversidad y la estructura del bosque, y puede ser utilizado para interpretar aspectos de la dinámica del bosque y las poblaciones. En caso de volver a efectuar las mediciones, es posible también obtener datos para desarrollar modelos. Sin embargo, los inventarios de los abundantes individuos del sotobosque requieren de mucho tiempo, y para propósitos de manejo forestal podría ser más sensato concentrarse en el muestreo de las especies a manejar, ya que, dentro del contexto del manejo forestal, sería probablemente más deseable el conocimiento específico de su ecología. 


\section{RECONOCIMIENTOS}

Arístides Vásquez, Nitzen Saavedra, David Maytahuari y Julio Irarica por su ayuda en el trabajo de campo. El Centro de Investigaciones Jenaro Herrera (CIJH), administrado por el Instituto de Investigaciones de la Amazonía Peruana (IIAP), por contribuir amablemente con sus instalaciones de campo y el apoyo logístico. Luis Freitas, Juan Ruiz y Anders Ræbild, por su ayuda en las identificaciones de campo y el establecimiento de las parcelas. Henning Christensen por su ayuda en la identificación de las muestras botánicas en la Universidad de Aarhus (AAU). Lars Peter Kvist por el apoyo durante todo el estudio. Los siguientes taxónomos gratamente ayudaron a identificar los especímenes botánicos: C.C. Berg, J. Brandbyge, B.B. Klitgard, G.P. Lewis, P.J.M. Mass, T.D. Pennington, G.T. Prance, H. Rainer, S.S. Renner, M. De Rico-Arce y H. Van der Werff. El apoyo financiero fue provisto por Dalhof Larsen y Horneman A/S, Trælasthandlerunionen y la Agencia Danesa para el Desarrollo Internacional (DANIDA). 


\section{BIBLIOGRAFÍA}

ALDES, D., SYNNOTT, T.J. 1992. Permanet sample plot techniques for mixed tropical forest. Oxford Forestry Institute, Oxford. Tropical Forestry Papers. no. $25.124 \mathrm{pp}$.

ANDERSEN, M.K. 1995. Jorde i peruviansk Amazonas. Thesis. Royal Veterinary and Agricultural University. 46 pp.

AYRES, J.M. 1995. As matas de várzea do mamirauá. MCT-CNP q Sociedade Civil Mamiraua, 123 pp.

BALSLEV, H., LUTEYN, J., OLLGAARD, B. HOLM-NIELSEN, L.B. 1987. Composition and structure of adjacent unflooded and floodplain forest in Amazonian Ecuador. Opera Botanica, 92: 37-57.

BONGERS, F., POPMA, J. DEL CASTILLO, J.M., CARABIAS, J. 1988. Structure and floristic composition of the lowland rain forest of Los Tuxtlas, Mexico. Vegetatio, 74: 55-80.

BOMM, B.M 1986. A forest inventory in Amazonian Bolivia. Biotropica, 18(4): 287-294.

BRUNIG, E.F. 1983. Vegetation structure and growth. In: F.B. Golley (Editor), Tropical rain forest ecossystems. Structure and function. Elsevier, Amsterdam, pp. 49-75.

CAMPBELl, D.G. DOUGLAS, C.D., PRANCE, G.T. MACIEL, U.N. 1986. Quantitative ecological inventory of terra firme and varzea tropical forest on the Rio Xingu, Brazilian Amazon. Brittonia, 38(4): 369-393.

CLARK, D.A., CLARK, D.B. 1992. Life history diversity of canopy and emergent trees in a neotropical rain forest. Ecological Monographs, 62(3): 315-344.

COLONNELLO, G. 1990. A Venezuelan floodplain study on the Orinoco River. Forest Ecology and Management, 33/34: 103-124.

CURTIS, J.T., MCINTOSH, R.P. 1950. The interrelations of certain analytic and synthetic phytosociological characters. Ecology, 31(3): 435-455. 
CURTIS, J.T., MCINTOSH, R.P. 1951. An upland forest continuum in the prairieforest border region of Wisconsin. Ecology, 32(3): 476-496.

DENSLOW, J.S. 1980. Grap partitioning among tropical rainforest trees. Biotropica, 12 (Suppl.): 47-55.

FOSTER, R.B., ARCE, J.B., WACHTER, T.S. 1986. Dispersal and the sequential plant communities in Amazonian Peru floodplain. In: A. Estrada, T.H. Fleming (Editor), Frugivores and seed dispersal. Dr W. Junk Publishers, Dordrecht, pp. 357-370.

FREITAS, L.A. 1996. Caracterización floristic y estructural de cuatro comunidades boscosas de la llanura aluvial inundable en la zona Jenaro Herrera, Amazonía Peruana. Instituto de Investigaciones de la amazonia Peruana, Iquitos. Documento Tecnico. no. $21.73 \mathrm{pp}$.

FREITAS, L.A. 1996. Caracterización floristic y estructural de cuatro comunidades boscosas de terraza baja en la zona de Jenaro Herrera, Amazonía Peruana. Instituto de Investigaciones de la amazonia Peruana, Iquitos. Documento Tecnico. no. 26. 77 pp.

GENTRY, A.H. DODSON, C. 1987. Contribucion of nontrees to species richness of a tropical rain forest. Biotropica, 19(2): 149-156.

GENTRY, A.H. EMMONS, L.H. 1987. Geographical variation in fertility, phenology, and composition of the understorey of neotropical forest. Biotropica, 19(3): 216-227.

GENTRY, A.H. TERBORGH, J. 1990. Composition and dynamcis of the Cocha Cashu "mature" floodplain forest. In: A.H. Gentry (Editor), Four neotropica rainforests. Yale University Press, New Haven and London, pp. 542-563.

GOMEZ-POMPA, A., BURLEY, F.W. 1991. The management of natural tropical forests. In: A. Gomez-Pompa, T.C. Whitmore, M. Hadley (Editor), Rain forest regeneration and management. UNESCO, Paris, pp. 3-18.

GREIG-SMITH, P. 1983. Quantitative plant ecology. Blackwell Scientific Publications, $359 \mathrm{pp}$. 
HUBBELL, S.P., FOSTER, R.B. 1992. Short-term dynamics of a neotropical forest: why wcological research matters to tropical conservation and management. OIKOS, 63: 48-61.

JORDAN, C.F. 1985. Nutrient cycling in tropical forest forest ecosystems. Wiley, Chichester.

KVIST, L.P., NEBEL, G. 1999. A review of Peruvian flood plain forests: Ecosystems, inhabitants and resource use. Royal Veterinary and Agricultural University, Department of Economics and Natural Resources, Unit of Forestry. Copenhagen. Unpublished manuscript.

LAMPRECHT, H. 1989. Silviculture in the tropics. Tropical forest ecosystems and their tree species - possibilites and methods for their long-term utilization. GTZ, Eschborn.

MORI, S.A., BOOM. B.M., DE CARVALHO, A.M., DOS SANTOS, T.S. 1983. Southern Bahian moist forests. The Botanical Review, 49(2): 155-232.

NEBEL, G., KVIST, L.P. VANCLAY, J.K., CHRISTENSEN, H., FREITAS. L., RUIZ, J. 1999 a. Structure and floristic composition of flood plain forests in the Peruvian Amazon: I. Overstorey. Royal Veterinary and Agricultural University, Department of Economics and Narutal Resources, Unit of Forestry. Copenhagen. Unpublished manuscript.

NEBEL, G., DRAGSTED, J. SIMONSEN, T.R., VANCLAY, J.K. 1999b. The Amazon flood palin forest tree Maquira coriacea ( Karsten ) C.C. Berg: Aspects of ecology and management Royal Veterinary and Agricultural University, Department of Economics and Narutal Resources, Unit of Forestry. Copenhagen. Unpublished manuscript.

RANKIN-DE-MÉRONA, J.M. PRANCE, G.T. HUTCHINGS, R.W., SILVA, M.F. RODRIGUES, W.A., UEHLING, M.E. 1992. Preliminary results of a largescale tree inventory of uplan rain forest in the central Amazon. Acta Amazonica, 22 (4): 493-534.

RICHARDS, P.W. 1996. The tropical rain forest. Cambridge University Press, Cambridge, $575 \mathrm{pp}$. 
SALO, J., KALLIOLA, R., HAKKINEN, I., MAKINEN, Y., NIEMELA, P., PUHAKKA, M. COLEY, P.D. 1986. River dynamics and the diversity of Amazon lowland forest. Nature, 322: 254-258.

SWAINE, M.D. HALL, J.B., ALEXANDER, I.J. 1987. Tree pupulation dynamics at Khade, Ghana (1968-1982). Journal of Tropical Ecology, 3: 331-345.

SWAINE, M.D. WHITMORE, T.C. 1988. On the definition of ecological species groups in tropical rain forest. Vegetation, 75: 81-86.

SORENCE, T. 1948. A methold of establishing groups of equal amplitude in plant sociology based on similarity of species content and its application to analyses of the vegetation on Danish commons. Det Kongelige Danske Videnskabers Selskab, Biologiske Skrifter, 5(4): 1-34.

UHL, C., MURPHY, P.G. 1981. Composition, structure, and regeneration of a tierra firme forest in the Amazon basin of Venezuela. Tropical Ecology, 22(2): 219-237.

VALENCIA, R., BALSLEV, H., PAZ Y MINO, G.C. 1994. High tree alpha-diversity in Amazonian Ecuador. Biodiversity and Conservation, 3: 21-28.

WHITMORE, T.C. 1995. Perspectives in tropical rain forest research. In: A.E. Lugo, C. Lowe (Editor), Tropical forests: Ecology and management. SpringerVerlag, Berlin, pp. 397-407.

WHITMORE, T.C., BROWN, N.D. SWAINE, M.D. KENEDY, D., GOODWINBAILEY, C.L., GONG, W.K. 1983. Secondary succession from seed in tropical rain forests. Forestry abstracts, 44 (12): 767-779.

WORBES, M. 1983. Vegetationskundliche Untersuchunger zweier Uberschwemmungswalder in Zentralamazonien - vorlaufige Ergebnisse. Amazonía, 8(1): 47-65.

WORBES, M. 1986. Lebensbedingungen und Holzwachstum in zentralamazonischen Uberschwemmungswaldern. Scripta Geobotanica, 17: 7-112.

WORBES, M. KLINGE, H., REVILLA, J.D., MARTIUS, C. 1992. On the dynamics, floristic subdivision and geographical distribution of várzea forests in Central Amazonía. Journal of Vegetation Science, 3: 553-564. 
Cuadro1. Número de familias, número de especies, número de individuos y áreas basales para los árboles de sotobosque en seis parcelas de una hectárea. En la parcela 1 cinco subparcelas de 16 x 16 m fueron inventariadas ( $1280 \mathrm{~m}^{2}$ ), mientras que en las otras parcelas se muestraron cuatro parcelas de $16 \times 16 \mathrm{~m}\left(1024 \mathrm{~m}^{2}\right)$. Las parcelas 1,2 y 3 pertenecen al bosque de restinga alta, mientras que las parcelas numeradas del 4 al 6 se localizan en el bosque de restinga baja.

\begin{tabular}{|c|c|c|c|c|}
\hline & $\begin{array}{c}\mathrm{N}^{\circ} \text { de } \\
\text { Familias }\end{array}$ & $\begin{array}{c}\mathrm{N}^{\circ} \text { de } \\
\text { Especies }\end{array}$ & $\begin{array}{c}\text { Individuos } \\
\text { por ha }\end{array}$ & $\begin{array}{c}\text { Area basal } \\
\mathrm{m}^{2} / \mathrm{ha}\end{array}$ \\
\hline Restinga alta & 45 & 160 & 4585 & 5,1 \\
\hline Parcela 1 & 43 & 121 & 4609 & 5,1 \\
\hline Parcela 2 & 35 & 87 & 4268 & 5,0 \\
\hline Parcela 3 & 39 & 109 & 4873 & 5,2 \\
\hline Restinga baja & 45 & 166 & 4320 & 5,0 \\
\hline Parcela 4 & 38 & 97 & 5049 & 5,7 \\
\hline Parcela 5 & 41 & 107 & 3740 & 4,3 \\
\hline Parcela 6 & 38 & 99 & 4170 & 4,9 \\
\hline Todos las parcelas & 47 & 240 & 4458 & 5,0 \\
\hline
\end{tabular}

Cuadro2. Números (superiores derecho) y porcentajes (inferior izquierdo) de las especies compartidas entre pares de parcelas. Los númercs reales de especies están entre paréntesis.

\begin{tabular}{|l|c|r|r|r|r|r|r|}
\hline \multirow{2}{*}{} & \multirow{2}{*}{ Parcela } & \multicolumn{3}{|c|}{ Restinga Alta } & \multicolumn{3}{|c|}{ Restinga Baja } \\
\cline { 3 - 8 } & & 1 & 2 & 3 & 4 & 5 & \multicolumn{1}{c|}{6} \\
\hline Restinga alta & 1 & $(121)$ & 71 & 78 & 65 & 72 & 52 \\
\hline & 2 & $47 \%$ & $(87)$ & 59 & 51 & 54 & 42 \\
\hline & 3 & $54 \%$ & $46 \%$ & $(109)$ & 69 & 70 & 59 \\
\hline Restinga baja & 4 & $36 \%$ & $31 \%$ & $41 \%$ & $(97)$ & 64 & 54 \\
\hline & 5 & $38 \%$ & $31 \%$ & $39 \%$ & $37 \%$ & $(107)$ & 62 \\
\hline & 6 & $25 \%$ & $23 \%$ & $32 \%$ & $30 \%$ & $34 \%$ & $(99)$ \\
\hline
\end{tabular}


ESTRUCTURA Y COMPOSICIÓN FLORÍSTICA DEL BOSQUE DE LA LLANURA ALUVIAL

Cuadro3. Número y porcentaje de especies que ocurren ya sea en bosques de restinga baja o alta, o en ambos bosques. Coeficientes de similitud de Jaccard y Sorensen.

\begin{tabular}{|l|r|r|r|r|}
\hline & \multicolumn{1}{|c|}{$\begin{array}{c}\text { Restinga } \\
\text { alta solo }\end{array}$} & $\begin{array}{c}\text { Restinga } \\
\text { baja solo }\end{array}$ & $\begin{array}{c}\text { Ambos } \\
\text { bosques }\end{array}$ & \multicolumn{1}{c|}{ Total } \\
\hline Número de especies & 38 & 44 & 122 & 204 \\
\hline$\%$ total de especies & 19 & 21 & 60 & 100 \\
\hline Coeficiente de Jaccard & - & - & 0,60 & - \\
\hline Coeficiente de Sorensen & - & - & 0,75 & - \\
\hline
\end{tabular}

Cuadro4. Valor de importancia de la familia (FIV) de las familias presentes en el sotobosque de los bosques de restinga alta y baja. FIV es la suma de la densidad relativa (rel. den.), diversidad relativa (rel. div .) y la dominancia relativa (rel. dom.) de cada familia.

\begin{tabular}{|l|r|r|r|r|r|r|r|r|}
\hline & \multicolumn{4}{|c|}{ Restinga Alta } & \multicolumn{3}{c|}{ Restinga Baja } \\
\cline { 2 - 10 } & $\begin{array}{r}\text { Rel. } \\
\text { den. }\end{array}$ & $\begin{array}{c}\text { Rel. } \\
\text { fre. }\end{array}$ & $\begin{array}{c}\text { Rel. } \\
\text { dom. }\end{array}$ & FIV & $\begin{array}{c}\text { Rel. } \\
\text { den. }\end{array}$ & $\begin{array}{c}\text { Rel. } \\
\text { fre. }\end{array}$ & $\begin{array}{c}\text { Rel. } \\
\text { dom. }\end{array}$ & FIV \\
\hline Anacardiaceae & 0,26 & 1,24 & 0,46 & 1,96 & 0,15 & 1,2 & 0,01 & 1,36 \\
\hline Annonaceae & 7,4 & 7,45 & 6,81 & 21,67 & 4,75 & 7,19 & 8,01 & 19,94 \\
\hline Apocynaceae & 0,66 & 1,24 & 0,49 & 2,39 & 0,3 & 1,2 & 0,43 & 1,93 \\
\hline Arecaceae & 6,88 & 2,48 & 8,56 & 17,92 & 4,3 & 1,2 & 2,78 & 8,27 \\
\hline Bombacaceae & 2,16 & 1,86 & 3,49 & 7,52 & 0,6 & 0,6 & 1,17 & 2,38 \\
\hline Boraginaceae & 0,92 & 1,24 & 1,37 & 3,53 & 0,53 & 1,2 & 1,14 & 2,86 \\
\hline Burseraceae & 2,03 & 0,62 & 2,35 & 5,01 & 0,45 & 0,6 & 0,34 & 1,39 \\
\hline Caesalpiniaceae & 0,26 & 1,24 & 0,37 & 1,88 & 0,53 & 1,2 & 0,57 & 2,29 \\
\hline Capparaceae & 0,92 & 0,62 & 1,33 & 2,87 & 0,53 & 0,6 & 0,9 & 2,03 \\
\hline Cecropiaceae & 0,66 & 1,86 & 1,8 & 4,32 & 0,98 & 0,6 & 0,63 & 2,21 \\
\hline Celastraceae & 0,13 & 0,62 & 0,13 & 0,88 & 0,53 & 1,2 & 0,16 & 1,88 \\
\hline Chrysobalanaceae & 1,11 & 2,48 & 0,48 & 4,08 & 0,9 & 4,19 & 1,55 & 6,65 \\
\hline Clusiaceae & 3,67 & 1,86 & 2,18 & 7,71 & 2,79 & 1,8 & 1,42 & 6 \\
\hline Combretaceae & 0,2 & 0,62 & 0,67 & 1,48 & 0,6 & 1,8 & 0,79 & 3,19 \\
\hline Dichapetalaceae & 0,13 & 0,62 & 0,03 & 0,78 & 0,08 & 0,6 & 0,01 & 0,68 \\
\hline Ebenaceae & 0,13 & 1,24 & 0,19 & 1,56 & 0,38 & 0,6 & 0,48 & 1,46 \\
\hline Elaeocarpaceae & 0,52 & 0,62 & 1,02 & 2,16 & 0,68 & 1,2 & 1,39 & 3,27 \\
\hline Euphorbiaceae & 6,09 & 5,59 & 6,57 & 18,26 & 9,8 & 5,99 & 11,33 & 27,12 \\
\hline Fabaceae & 1,51 & 4,97 & 1,66 & 8,13 & 1,73 & 4,19 & 1,53 & 7,45 \\
\hline Flacourtiaceae & 1,51 & 2,48 & 1,13 & 5,12 & 3,69 & 4,19 & 3,66 & 11,54 \\
\hline Icacinaceae & - & - & - & - & 0,23 & 0,6 & 0,74 & 1,57 \\
\hline Lauraceae & 4,06 & 4,97 & 3,72 & 12,75 & 3,54 & 5,39 & 5,6 & 14,53 \\
\hline
\end{tabular}

continúa... 


\begin{tabular}{|l|r|r|r|r|r|r|r|r|}
\hline Lecythidaceae & 1,38 & 2,48 & 1,74 & 5,62 & 2,19 & 2,4 & 2 & 6,58 \\
\hline Malpighiaceae & 0,07 & 0,62 & 0,04 & 0,73 & 0,6 & 0,6 & 0,3 & 1,5 \\
\hline Melastomataceae & 5,05 & 3,73 & 2,04 & 10,81 & 5,65 & 2,4 & 4,77 & 12,82 \\
\hline Meliaceae & 1,05 & 2,48 & 1,75 & 5,28 & 0,9 & 2,99 & 2 & 5,9 \\
\hline Mimosaceae & 3,8 & 5,59 & 5,14 & 14,53 & 3,99 & 5,39 & 8,37 & 17,76 \\
\hline Moraceae & 11,14 & 6,83 & 11,87 & 29,84 & 2,56 & 4,19 & 3,62 & 10,37 \\
\hline Myristicaceae & 1,31 & 1,86 & 1,34 & 4,51 & 3,09 & 1,8 & 4,36 & 9,24 \\
\hline Myrsinaceae & 0,26 & 0,62 & 0,28 & 1,16 & 0,98 & 0,6 & 0,72 & 2,3 \\
\hline Myrtaceae & 1,97 & 4,97 & 2,48 & 9,42 & 5,88 & 7,19 & 6,74 & 19,81 \\
\hline Nyctaginaceae & 1,38 & 0,62 & 1,33 & 3,33 & 1,88 & 1,2 & 1,93 & 5,01 \\
\hline Ochnaceae & 0,13 & 0,62 & 0,02 & 0,77 & 0,3 & 0,6 & 0,11 & 1,01 \\
\hline Olacaceae & 6,62 & 1,86 & 1,66 & 10,14 & 10,1 & 2,4 & 2,78 & 15,28 \\
\hline Passifloraceae & 0,07 & 0,62 & 0,01 & 0,69 & - & - & - & - \\
\hline Polygonaceae & 1,18 & 2,48 & 1,46 & 5,12 & 2,11 & 2,4 & 2,4 & 6,91 \\
\hline Quiinaceae & 0,13 & 0,62 & 0,01 & 0,77 & 0,15 & 0,6 & 0,1 & 0,85 \\
\hline Rubiaceae & 7,34 & 6,83 & 3,27 & 17,44 & 10,40 & 8,38 & 4,35 & 23,13 \\
\hline Sapindaceae & 1,25 & 1,24 & 1,1 & 3,59 & 1,28 & 2,4 & 0,85 & 4,53 \\
\hline Sapotaceae & 2,56 & 3,11 & 2,28 & 7,94 & 2,56 & 1,8 & 3,95 & 8,31 \\
\hline Simaroubaceae & 0,46 & 1,24 & 0,02 & 1,72 & 0,23 & 1,2 & 0,12 & 1,55 \\
\hline Solanaceae & 1,05 & 1,86 & 0,24 & 3,15 & 3,24 & 1,2 & 0,75 & 5,18 \\
\hline Sterculiaceae & 0,72 & 1,86 & 0,9 & 3,49 & 0,15 & 0,6 & 0,07 & 0,82 \\
\hline Theophrastaceae & 0,33 & 0,62 & 0,04 & 0,99 & - & - & - & - \\
\hline Tiliaceae & - & - & - & - & 0,6 & 1,2 & 1,22 & 3,02 \\
\hline Violaceae & 8,85 & 1,24 & 15,61 & 25,7 & 0,98 & 1,2 & 1,97 & 4,15 \\
\hline Unidentified & 0,72 & 0,00 & 0,54 & 1,26 & 2,11 & 0 & 1,87 & 3,98 \\
\hline
\end{tabular}

Cuadro5. Número de especies identificadas en el estrato superior (individuas de más de 10 cm DAP), en el sotobosque (individuos mayores a 1,5 m y hasta 10 cm de DAP), y el común para sotobosque y estrato superior de bosques de restinga (alta y baja).

\begin{tabular}{|c|c|r|r|r|r|r|}
\hline & Total & $\begin{array}{c}\text { Bosque alto } \\
\text { total }\end{array}$ & $\begin{array}{c}\text { Sotobosque } \\
\text { total }\end{array}$ & $\begin{array}{c}\text { Bosque alto } \\
\text { total }\end{array}$ & $\begin{array}{c}\text { Sotobosque } \\
\text { solo }\end{array}$ & Común \\
\hline Restinga Alta & 205 & $139(68 \%)$ & $160(78 \%)$ & $45(22 \%)$ & $66(32 \%)$ & $94(46 \%)$ \\
\hline Parc. 1 & 149 & $86(58 \%)$ & $120(81 \%)$ & $29(20 \%)$ & $63(42 \%)$ & $57(38 \%)$ \\
\hline Parc. 2 & 134 & $98(73 \%)$ & $87(65 \%)$ & $47(35 \%)$ & $36(27 \%)$ & $51(38 \%)$ \\
\hline Parc. 3 & 150 & $97(64 \%)$ & $109(73 \%)$ & $41(28 \%)$ & $53(35 \%)$ & $56(37 \%)$ \\
\hline Restinga Baja & 228 & $181(79 \%)$ & $166(73 \%)$ & $62(27 \%)$ & $47(21 \%)$ & $119(52 \%)$ \\
\hline Parc. 4 & 156 & $120(77 \%)$ & $97(81 \%)$ & $59(38 \%)$ & $36(23 \%)$ & $61(39 \%)$ \\
\hline Parc. 5 & 173 & $131(76 \%)$ & $107(82 \%)$ & $66(38 \%)$ & $42(24 \%)$ & $65(38 \%)$ \\
\hline Parc. 6 & 171 & $129(74 \%)$ & $99(58 \%)$ & $72(42 \%)$ & $42(25 \%)$ & $57(33 \%)$ \\
\hline Todas las parcelas & 264 & $208(79 \%)$ & $204(76 \%)$ & $60(23 \%)$ & $56(21 \%)$ & $148(56 \%)$ \\
\hline
\end{tabular}


APÉNDICE 1. Valor de importancia de la especie (SIV) para las especies presentes en la restinga alta y baja, así como en ambos bosques. SIV es la suma de la densidad relativa (rel. den), frecuencia relativa (rel. fre) y la dominancia relativa (rel.dom) de cada especie. Especies características en ambos bosques de restinga (R), de restinga alta $(\mathrm{H})$ y de restinga baja (L). Los totales absolutos en el final de las columnas permiten el cálculo de los valores absolutos para cada especie. Los números después de los nombres de las especies son números de colección de J. Ruiz et al. (solamente el número) y Nebel (con "N"), tal como están registrados en la Universidad de Aarhus (AAU), Dinamarca.

\begin{tabular}{|c|c|c|c|c|c|c|c|c|c|}
\hline & \multirow{2}{*}{$\begin{array}{l}\text { Caract. } \\
\text { espec. }\end{array}$} & \multicolumn{4}{|c|}{ Restinga Alta } & \multicolumn{4}{|c|}{ Restinga Baja } \\
\hline & & $\begin{array}{l}\text { Rel. } \\
\text { den. }\end{array}$ & $\begin{array}{l}\text { Rel. } \\
\text { fre. }\end{array}$ & $\begin{array}{l}\text { Rel. } \\
\text { dom. }\end{array}$ & SIV & $\begin{array}{l}\text { Rel. } \\
\text { dem. }\end{array}$ & $\begin{array}{l}\text { Rel. } \\
\text { fre. }\end{array}$ & $\begin{array}{l}\text { Rel. } \\
\text { dom. }\end{array}$ & SIV \\
\hline \multicolumn{10}{|l|}{ ANACARDIACEAE } \\
\hline Spondias mombin L. 2278 & & 0,26 & 0,6 & 0,46 & 1,32 & - & - & - & - \\
\hline Tapirira guianensis Aublet 1659 & & - & - & - & - & 0,15 & 0,34 & 0,01 & $\mathbf{0 , 5}$ \\
\hline \multicolumn{10}{|l|}{ ANNONACEAE } \\
\hline Crematosperma sp. 8712 & $\mathrm{~L}$ & 0,72 & 1,2 & 0,65 & 2,57 & 1,06 & 1,87 & 1,8 & 4,72 \\
\hline Duguetia spixiana C. Martius 5508 & & 0,26 & 0,6 & 0,39 & 1,25 & 0,15 & 0,34 & 0,23 & $\mathbf{0 , 7 2}$ \\
\hline Duguetia sp. N307038 & & 0,07 & 0,15 & 0,02 & $\mathbf{0 , 2 3}$ & - & - & - & - \\
\hline Guatteria sp. 12006 & $\mathrm{H}$ & 1,44 & 1,2 & 0,65 & 3,3 & 0,15 & 0,34 & 0,22 & 0,71 \\
\hline Guatteria sp. 35202 & & - & - & - & - & 0,08 & 0,17 & 0,47 & 0,72 \\
\hline Guatteria sp. 4 N807332 & & 0,39 & 0,75 & 0,35 & 1,5 & 0,08 & 0,17 & 0,1 & 0,34 \\
\hline Malmea sp. 6013 & & 0,2 & 0,45 & 0,49 & 1,14 & 0,15 & 0,34 & 0,31 & $\mathbf{0 , 8}$ \\
\hline $\begin{array}{l}\text { Oxandra sphaerocarpa } \\
\text { R. E. Fries } 1579\end{array}$ & $\mathrm{R}$ & 1,18 & 1,35 & 1,19 & 3,72 & 0,9 & 1,36 & 2,43 & 4,69 \\
\hline $\begin{array}{l}\text { Pseudoxandra polyphleba } \\
\text { (Diels) R. E. Fries } 4085\end{array}$ & & 0,26 & 0,45 & 0,07 & $\mathbf{0 , 7 8}$ & 0,08 & 0,17 & 0,27 & $\mathbf{0 , 5 2}$ \\
\hline Rollinia cuspidata C. Martius 9266 & $\mathrm{~L}$ & 0,85 & 1,2 & 0,65 & 2,7 & 0,98 & 1,36 & 0,98 & 3,32 \\
\hline Unonopsis floribunda Diels 1266 & & 0,66 & 1,2 & 0,72 & 2,58 & 0,3 & 0,68 & 0,4 & 1,38 \\
\hline Xylopia micans R. E. Fries 1165 & & 0,72 & 1,05 & 0,85 & 2,62 & 0,6 & 1,19 & 0,55 & 2,34 \\
\hline Xylopia sp. 12024 & & 0,66 & 1,2 & 0,78 & 2,63 & 0,23 & 0,51 & 0,25 & 0,98 \\
\hline \multicolumn{10}{|l|}{ APOCYNACEAE } \\
\hline $\begin{array}{l}\text { Himatanthus bracteatus } \\
\text { (A. DC.) Woodson } 2048\end{array}$ & & 0,07 & 0,15 & 0,08 & 0,3 & 0,23 & 0,34 & 0,35 & 0,91 \\
\hline $\begin{array}{l}\text { Tabernaemontana markgrafiana } \\
\text { J. F. Macbride N209199 }\end{array}$ & & 0,59 & 0,75 & 0,4 & 1,74 & 0,08 & 0,17 & 0,08 & $\mathbf{0 , 3 3}$ \\
\hline \multicolumn{10}{|l|}{ ARECACEAE } \\
\hline Astrocaryum jauari C. Martius & & 0,07 & 0,15 & 0,12 & $\mathbf{0 , 3 3}$ & - & - & - & - \\
\hline Bactris sp. & $\mathrm{R}$ & 3,28 & 1,35 & 2,01 & 6,63 & 4,14 & 1,02 & 2,5 & 7,67 \\
\hline Euterpe precatoria $\mathrm{C}$. Martius & & 0,07 & 0,15 & 0,26 & 0,48 & 0,15 & 0,17 & 0,27 & 0,6 \\
\hline
\end{tabular}




\begin{tabular}{|c|c|c|c|c|c|c|c|c|c|}
\hline Scheelea brachyclada Burret & $\mathrm{H}$ & 3,47 & 1,8 & 6,17 & 11,44 & - & - & - & - \\
\hline \multicolumn{10}{|l|}{ BОМВАСАCEAE } \\
\hline $\begin{array}{l}\text { Ceiba samauma (C. Martius \& } \\
\text { Zuccarini) Schumann } 5345\end{array}$ & & 0,13 & 0,3 & 0,18 & 0,61 & - & - & - & - \\
\hline Matisia bracteolosa Ducke 2277 & $\mathrm{H}$ & 1,9 & 1,5 & 3,01 & 6,41 & 0,6 & 0,68 & 1,17 & 2,46 \\
\hline Pachira aquatica Aublet 4535 & & 0,13 & 0,3 & 0,3 & $\mathbf{0 , 7 3}$ & - & - & - & - \\
\hline \multicolumn{10}{|l|}{ BORAGINACEAE } \\
\hline Cordia nodosa Lamarck 3049 & $\mathrm{H}$ & 0,85 & 1,35 & 1,37 & 3,57 & 0,45 & 0,85 & 1,13 & 2,43 \\
\hline Unidentified & & 0,07 & 0,15 & 0 & $\mathbf{0 , 2 2}$ & 0,08 & 0,17 & 0 & 0,25 \\
\hline \multicolumn{10}{|l|}{ BURSERACEAE } \\
\hline Protium nodulosum Swart 1090 & $\mathrm{H}$ & 2,03 & 1,65 & 2,35 & 6,04 & 0,45 & 0,85 & 0,34 & 1,64 \\
\hline \multicolumn{10}{|l|}{ CAESALPINIACEAE } \\
\hline Cynometra sp. 9060 & & 0,13 & 0,3 & 0,08 & $\mathbf{0 , 5 1}$ & 0,08 & 0,17 & 0,13 & $\mathbf{0 , 3 7}$ \\
\hline $\begin{array}{l}\text { Senna bacillaris var, Benthamiana } \\
\text { (J, F, Macbride) H, }\end{array}$ & & 0,13 & 0,3 & 0,29 & $\mathbf{0 , 7 2}$ & 0,45 & 0,68 & 0,44 & 1,57 \\
\hline \multicolumn{10}{|l|}{ Irwin \& Barneby 1439} \\
\hline \multicolumn{10}{|l|}{ CAPPARACEAE } \\
\hline Capparis sola J. F. Macbride 3013 & $\mathrm{H}$ & 0,92 & 1,05 & 1,33 & 3,3 & 0,53 & 1,02 & 0,9 & 2,45 \\
\hline \multicolumn{10}{|l|}{ CECROPIACEAE } \\
\hline $\begin{array}{l}\text { Cecropia ficifolia Warburg } \\
\text { ex Snethlage N907037 }\end{array}$ & & - & - & - & - & 0,98 & 0,85 & 0,63 & 2,46 \\
\hline Cecropia unidentified & & 0,13 & 0 & 0,12 & $\mathbf{0 , 2 5}$ & - & - & - & - \\
\hline $\begin{array}{l}\text { Pourouma acuminata } \text { C. Martius } \\
\text { ex Miquel } 1356\end{array}$ & & 0,07 & 0,15 & 0,37 & $\mathbf{0 , 5 8}$ & - & - & - & - \\
\hline $\begin{array}{l}\text { Pourouma cecropiifolia } \\
\text { C, Martius } 2014\end{array}$ & & 0,39 & 0,75 & 1,31 & 2,45 & - & - & - & - \\
\hline $\begin{array}{l}\text { Pourouma cucura } \\
\text { Standley \& Quatrecasas }\end{array}$ & & 0,07 & 0,15 & 0 & $\mathbf{0 , 2 2}$ & - & - & - & - \\
\hline \multicolumn{10}{|l|}{ CELASTRACEAE } \\
\hline $\begin{array}{l}\text { Maytenus macrocarpa } \\
\text { (R. \& P.) Briquet } 2408\end{array}$ & & 0,13 & 0,3 & 0,13 & 0,56 & 0,15 & 0,34 & 0,14 & 0,63 \\
\hline Maytenus sp. N907022 & & - & - & - & - & 0,38 & 0,51 & 0,02 & $\mathbf{0 , 9 1}$ \\
\hline \multicolumn{10}{|l|}{ CHRYSOBALANACEAE } \\
\hline Couepia subcordata Bentham 9400 & & - & - & - & - & 0,08 & 0,17 & 0,1 & $\mathbf{0 , 3 5}$ \\
\hline Couepia sp. 6389 & & 0,13 & 0,3 & 0,01 & 0,44 & 0,15 & 0,34 & 0,22 & 0,71 \\
\hline $\begin{array}{l}\text { Hirtella triandra } \mathrm{sp.} \\
\text { Triandra Swarts } 3243\end{array}$ & & 0,46 & 0,6 & 0,06 & 1,11 & 0,15 & 0,34 & 0,04 & $\mathbf{0 , 5 3}$ \\
\hline Licania britteniana Fritsch 3088 & & 0,26 & 0,6 & 0,25 & 1,12 & 0,3 & 0,51 & 0,7 & 1,51 \\
\hline $\begin{array}{l}\text { Licania macrocarpa } \\
\text { Cuatrecasas } 2581\end{array}$ & & - & - & - & - & 0,08 & 0,17 & 0,25 & 0,5 \\
\hline Licania micrantha Miquel 5558 & & - & - & - & - & 0,08 & 0,17 & 0,06 & $\mathbf{0 , 3 1}$ \\
\hline $\begin{array}{l}\text { Parinari parilis J.F. } \\
\text { Macbride } 1170\end{array}$ & & 0,26 & 0,6 & 0,17 & 1,03 & 0,08 & 0,17 & 0,17 & $\mathbf{0 , 4 2}$ \\
\hline CLUSIACEAE & & & & & & & & & \\
\hline
\end{tabular}


ESTRUCTURA Y COMPOSICIÓN FLORÍSTICA DEL BOSQUE DE LA LLANURA ALUVIAL

\begin{tabular}{|c|c|c|c|c|c|c|c|c|c|}
\hline $\begin{array}{l}\text { Garcinia macrophylla } \\
\text { C. Martius } 7349\end{array}$ & $\mathrm{~L}$ & 1,18 & 0,6 & 0,65 & 2,43 & 2,34 & 2,04 & 1,27 & 5,65 \\
\hline $\begin{array}{l}\text { Garcinia madruno } \\
\text { (Kunth) Hammel } 8113\end{array}$ & $\mathrm{H}$ & 2,42 & 1,8 & 1,51 & 5,73 & 0,38 & 0,68 & 0,14 & 1,2 \\
\hline Tovomita sp. 6773 & & - & - & - & - & 0,08 & 0,17 & 0 & 0,25 \\
\hline Vismia angusta Miquel 1298 & & 0,07 & 0,15 & 0,02 & $\mathbf{0 , 2 3}$ & - & - & - & - \\
\hline \multicolumn{10}{|l|}{ COMBRETACEAE } \\
\hline $\begin{array}{l}\text { Buchenavia amazonia } \\
\text { Al-Mayah \& Stace } 6316\end{array}$ & & - & - & - & - & 0,08 & 0,17 & 0,07 & $\mathbf{0 , 3 2}$ \\
\hline $\begin{array}{l}\text { Terminalia dichotoma } \\
\text { G. Meyer } 5598\end{array}$ & & - & - & - & - & 0,08 & 0,17 & 0,01 & 0,26 \\
\hline $\begin{array}{l}\text { Terminalia oblonga (Ruiz Lopez \& } \\
\text { Pavon) Steudel } 2196\end{array}$ & & 0,2 & 0,45 & 0,67 & 1,31 & 0,45 & 0,85 & 0,71 & 2,01 \\
\hline \multicolumn{10}{|l|}{ DICHAPETALACEAE } \\
\hline Tapura sp. 5440 & & 0,13 & 0,3 & 0,03 & 0,46 & 0,08 & 0,17 & 0,01 & 0,25 \\
\hline \multicolumn{10}{|l|}{ EBENACEAE } \\
\hline Diospyros sp. 18716 & & 0,07 & 0,15 & 0,05 & $\mathbf{0 , 2 7}$ & 0,38 & 0,85 & 0,48 & 1,71 \\
\hline Diospyros sp. 5 & & 0,07 & 0,15 & 0,14 & $\mathbf{0 , 3 5}$ & - & - & - & - \\
\hline \multicolumn{10}{|l|}{ ELAEOCARPACEAE } \\
\hline $\begin{array}{l}\text { Sloanea guianensis } \\
\text { (Aublet) Bentham } 6443\end{array}$ & & 0,52 & 0,9 & 1,02 & 2,44 & 0,6 & 0,85 & 1,36 & 2,81 \\
\hline Sloanea sp. 27201 & & - & - & - & - & 0,08 & 0,17 & 0,03 & 0,28 \\
\hline \multicolumn{10}{|l|}{ EUPHORBIACEAE } \\
\hline $\begin{array}{l}\text { Alchornea shomburgkii } \\
\text { Klotzsch } 6577\end{array}$ & & 0,2 & 0,45 & 0,12 & 0,76 & 0,45 & 1,02 & 0,86 & 2,33 \\
\hline Cleidion sp. & & - & - & - & - & 0,08 & 0,17 & 0,09 & $\mathbf{0 , 3 4}$ \\
\hline Croton cuneatus Klotzsch 3553 & & 0,66 & 0,45 & 0,63 & 1,74 & 1,51 & 1,19 & 0,84 & 3,53 \\
\hline $\begin{array}{l}\text { Drypetes amazonica var. Peruviana } \\
\text { J. F. Macbride } 2228\end{array}$ & $\mathrm{R}$ & 3,93 & 1,8 & 4,69 & 10,42 & 6,78 & 1,7 & 8,19 & 16,67 \\
\hline $\begin{array}{l}\text { Glycydendron amazonicum } \\
\text { Ducke } 7625\end{array}$ & & 0,2 & 0,3 & 0,17 & 0,67 & 0,08 & 0,17 & 0,07 & $\mathbf{0 , 3 2}$ \\
\hline Hura crepitans L. 2137 & & 0,13 & 0,3 & 0,19 & 0,62 & - & - & - & - \\
\hline $\begin{array}{l}\text { Jablonskia congesta (Bentham } \\
\text { ex Muell, Arg,) Webster N407513 }\end{array}$ & & 0,07 & 0,15 & 0,01 & 0,23 & 0,15 & 0,34 & 0,01 & 0,5 \\
\hline $\begin{array}{l}\text { Mabea nitida } \text { Spruce ex } \\
\text { Bentham } 5327\end{array}$ & & - & - & - & - & 0,08 & 0,17 & 0,1 & $\mathbf{0 , 3 5}$ \\
\hline Margaritaria nobilis L. f. N909168 & & - & - & - & - & 0,23 & 0,34 & 0,03 & $\mathbf{0 , 5 9}$ \\
\hline Podocalyx sp. 9484 & & 0,13 & 0,15 & 0,12 & 0,4 & 0,3 & 0,34 & 0,37 & $\mathbf{1 , 0 1}$ \\
\hline $\begin{array}{l}\text { Sapium glandulosum } \\
\text { (L.) Morong } 5342\end{array}$ & & 0,66 & 0,6 & 0,57 & 1,83 & 0,15 & 0,34 & 0,77 & 1,26 \\
\hline Sapium marmierii Huber 2211 & & 0,13 & 0,15 & 0,07 & $\mathbf{0 , 3 5}$ & - & - & - & - \\
\hline \multicolumn{10}{|l|}{ FABACEAE } \\
\hline $\begin{array}{l}\text { Andira inermis (W, Wright) } \\
\text { H.B.K. ex DC. } 5239\end{array}$ & & 0,2 & 0,3 & 0,15 & 0,65 & 0,15 & 0,17 & 0,04 & 0,36 \\
\hline Ormosia sp. L 3404 & & 0,13 & 0,3 & 0,34 & 0,77 & - & - & - & - \\
\hline
\end{tabular}




\begin{tabular}{|c|c|c|c|c|c|c|c|c|c|}
\hline Platymiscium stipulare Benth. 3382 & & 0,07 & 0,15 & 0,46 & 0,67 & 0,08 & 0,17 & 0,17 & 0,42 \\
\hline $\begin{array}{l}\text { Pterocarpus amazonum (C. Martius } \\
\text { ex Bentham) Amshoff } 7003\end{array}$ & & - & - & - & - & 0,23 & 0,34 & 0,05 & 0,61 \\
\hline Pterocarpus sp. 13172 & & 0,13 & 0,3 & 0,25 & 0,68 & 0,15 & 0,34 & 0,44 & $\mathbf{0 , 9 3}$ \\
\hline Pterocarpus sp. 28465 & & 0,07 & 0,15 & 0,01 & 0,22 & 0,23 & 0,51 & 0,03 & 0,76 \\
\hline $\begin{array}{l}\text { Swartzia cardiosperma } \\
\text { Spruce ex Bentham } 1152\end{array}$ & & 0,07 & 0,15 & 0,02 & 0,24 & 0,53 & 1,02 & 0,41 & 1,95 \\
\hline Swartzia simplex (Sprengel) 1258 & & 0,79 & 0,9 & 0,35 & 2,03 & 0,38 & 0,85 & 0,39 & 1,61 \\
\hline Swartzia sp. 6121 & & 0,07 & 0,15 & 0,08 & $\mathbf{0 , 3}$ & - & - & - & - \\
\hline \multicolumn{10}{|l|}{ FLACOURTIACEAE } \\
\hline Casearia aculeata Jacquin 1187 & $\mathrm{~L}$ & 0,66 & 0,45 & 0,27 & 1,37 & 1,06 & 1,19 & 0,92 & 3,16 \\
\hline $\begin{array}{l}\text { Casearia arborea } \\
\text { (Richard) Urban } 8431\end{array}$ & & 0,26 & 0,6 & 0,34 & 1,2 & 0,08 & 0,17 & 0,33 & $\mathbf{0 , 5 7}$ \\
\hline Casearia sylvestris Swartz 8320 & & 0,2 & 0,3 & 0,04 & 0,54 & 0,6 & 0,51 & 0,85 & 1,96 \\
\hline $\begin{array}{l}\text { Hasseltia floribunda } \\
\text { H.B.K. N209184 }\end{array}$ & & 0,39 & 0,75 & 0,48 & 1,62 & 0,08 & 0,17 & 0,06 & $\mathbf{0 , 3}$ \\
\hline $\begin{array}{l}\text { Laetia corymbulosa } \\
\text { Spruce ex Bentham } 9289\end{array}$ & $\mathrm{~L}$ & - & - & - & - & 1,73 & 1,02 & 1,2 & 3,96 \\
\hline Xylosma sp. 18569 & & - & - & - & - & 0,08 & 0,17 & 0,3 & $\mathbf{0 , 5 4}$ \\
\hline Xylosma sp. 2 & & - & - & - & - & 0,08 & 0,17 & 0 & 0,25 \\
\hline \multicolumn{10}{|l|}{ ICACINACEAE } \\
\hline Calatola venezuelana Pittier 4328 & & - & - & - & - & 0,23 & 0,34 & 0,74 & 1,31 \\
\hline \multicolumn{10}{|l|}{ LAURACEAE } \\
\hline Aniba guianensis Aublet 9172 & & - & - & - & - & 0,08 & 0,17 & 0,01 & 0,26 \\
\hline Aniba sp. 11138 & $\mathrm{H}$ & 1,11 & 1,35 & 1,23 & 3,69 & 0,6 & 0,68 & 1,23 & 2,51 \\
\hline $\begin{array}{l}\text { Endlicheria formosa } \\
\text { A.C. Smith } 2207\end{array}$ & & - & - & - & - & 0,08 & 0,17 & 0,13 & $\mathbf{0 , 3 8}$ \\
\hline Endlicheria sp. 7477 & & 0,13 & 0,3 & 0,06 & 0,49 & 0,45 & 0,51 & 0,16 & 1,12 \\
\hline Endlicheria verticillata Mez 9737 & & 0,07 & 0,15 & 0,01 & $\mathbf{0 , 2 2}$ & 0,23 & 0,51 & 4 & 1,13 \\
\hline $\begin{array}{l}\text { Nectandra cuneato - } \\
\text { cordata Mez } 6537\end{array}$ & & 0,59 & 0,6 & 0,77 & 1,96 & 0,38 & 0,68 & 0,29 & 1,35 \\
\hline Ocotea cernua (Nees) Mez 8437 & & 1,11 & 1,2 & 0,56 & 2,87 & 0,38 & 0,85 & 0,61 & 1,83 \\
\hline Ocotea javitensis 9056 & & 0,13 & 0,3 & 0,25 & 0,68 & 0,3 & 0,68 & 0,65 & 1,64 \\
\hline $\begin{array}{l}\text { Pleurothyrium parviflorum } \\
\text { Ducke } 1278\end{array}$ & $\mathrm{~L}$ & 0,59 & 1,05 & 0,61 & 2,25 & 0,98 & 1,36 & 1,95 & 4,29 \\
\hline No identificado sp. 4 & & 0,26 & 0,45 & 0,22 & $\mathbf{0 , 9 3}$ & - & - & - & - \\
\hline No identificado & & 0,07 & 0 & 0,01 & $\mathbf{0 , 0 8}$ & 0,08 & 0 & 0,17 & $\mathbf{0 , 2 4}$ \\
\hline \multicolumn{10}{|l|}{ LECYTHIDACEAE } \\
\hline Couroupita guianensis Aublet 7369 & & 0,07 & 0,15 & 0,08 & $\mathbf{0 , 3}$ & 0,15 & 0,34 & 0,18 & 0,67 \\
\hline $\begin{array}{l}\text { Couratari oligantha } \\
\text { A.C. Smith } 6337\end{array}$ & & 0,2 & 0,3 & 0,17 & $\mathbf{0 , 6 7}$ & 0,3 & 0,51 & 0,14 & 0,95 \\
\hline $\begin{array}{l}\text { Eschweilera parvifolia } \\
\text { C. Martius ex A. DC. } 5031\end{array}$ & $\mathrm{~L}$ & 0,33 & 0,75 & 0,19 & 1,26 & 1,36 & 1,87 & 1,18 & 4,4 \\
\hline $\begin{array}{l}\text { Eschweilera turbinata } \\
\text { (Berg) Niedenzu } 5019\end{array}$ & $\mathrm{H}$ & 0,79 & 1,05 & 1,31 & 3,15 & 0,38 & 0,51 & 0,5 & 1,39 \\
\hline
\end{tabular}


ESTRUCTURA Y COMPOSICIÓN FLORÍSTICA DEL BOSQUE DE LA LLANURA ALUVIAL

\begin{tabular}{|c|c|c|c|c|c|c|c|c|c|}
\hline MALPIGHIACEAE & & & & & & & & & \\
\hline $\begin{array}{l}\text { Byrsonima densa } \\
\text { (Poiret) DC. } 9104\end{array}$ & & 0,07 & 0,15 & 0,04 & 0,25 & 0,6 & 0,68 & 0,3 & 1,58 \\
\hline \multicolumn{10}{|l|}{ MELASTOMACEAE } \\
\hline $\begin{array}{l}\text { Miconia centrodesma } \\
\text { Wurdack } 8097\end{array}$ & & 0,2 & 0,3 & 0,17 & 0,67 & 0,08 & 0,17 & 0,24 & 0,49 \\
\hline Miconia sp. 13061 & & 0,39 & 0,75 & 0,6 & 1,74 & - & - & - & - \\
\hline Mouriri grandiflora A. DC. 9273 & $\mathrm{~L}$ & 1,31 & 0,75 & 0,69 & 2,75 & 1,73 & 1,87 & 3,57 & 7,17 \\
\hline Mouriri sp. & & 0,07 & 0,15 & 0,02 & 0,24 & - & - & - & - \\
\hline $\begin{array}{l}\text { Tococa coronata } \\
\text { Bentham N307114 }\end{array}$ & $\mathrm{L}$ & 0,85 & 0,9 & 0,19 & 1,94 & 1,81 & 1,7 & 0,71 & 4,21 \\
\hline Tococa sp. N109148 & $\mathrm{R}$ & 2,23 & 1,2 & 0,37 & 3,79 & 2,03 & 1,36 & 0,25 & 3,64 \\
\hline \multicolumn{10}{|l|}{ MELIACEAE } \\
\hline Cedrela odorata L. 2066 & & 0,2 & 0,45 & 0,54 & 1,19 & 0,08 & 0,17 & 0,16 & 0,41 \\
\hline Guarea macrophylla Vahl 3230 & & 0,46 & 0,6 & 0,69 & 1,75 & 0,38 & 0,85 & 0,96 & 2,19 \\
\hline $\begin{array}{l}\text { Trichilia inaequilatea } \\
\text { Penngton } 7021\end{array}$ & & - & - & - & - & 0,08 & 0,17 & 0,1 & 0,35 \\
\hline Trichilia pallida Swartz 3449 & & 0,13 & 0,3 & 0,43 & $\mathbf{0 , 8 6}$ & 0,08 & 0,17 & 0,07 & $\mathbf{0 , 3 1}$ \\
\hline $\begin{array}{l}\text { Trichilia pleeana (Adr. Jussieu) } \\
\text { C. DC. } 6037\end{array}$ & & 0,26 & 0,45 & 0,08 & $\mathbf{0 , 7 9}$ & - & - & - & - \\
\hline Trichilia rubra C. DC. 7191 & & - & - & - & - & 0,3 & 0,51 & 0,71 & 1,52 \\
\hline \multicolumn{10}{|l|}{ MIMOSACEAE } \\
\hline Inga bourgonii (Aublet) DC. 5569 & & 0,07 & 0,15 & 0,02 & $\mathbf{0 , 2 3}$ & 0,15 & 0,34 & 0,29 & 0,78 \\
\hline $\begin{array}{l}\text { Inga cinnamomea Spruce } \\
\text { ex Bentham } 1097\end{array}$ & $\mathrm{H}$ & 0,92 & 1,35 & 2,13 & 4,4 & 0,08 & 0,17 & 0,23 & $\mathbf{0 , 4 7}$ \\
\hline Inga nobilis Wildenow 1180 & & 0,26 & 0,45 & 0,6 & 1,31 & 0,08 & 0,17 & 0,4 & 0,64 \\
\hline Inga pavoniana $\mathrm{G}$. Don 4264 & & 0,46 & 0,3 & 0,19 & 0,95 & - & - & - & - \\
\hline Inga psittacorum L. Uribe 1515 & & - & - & - & - & 0,15 & 0,34 & 0,04 & $\mathbf{0 , 5 3}$ \\
\hline $\begin{array}{l}\text { Inga semialata (Vell. Conc.) } \\
\text { C. Martius } 1002\end{array}$ & & 0,13 & 0,3 & 0,43 & $\mathbf{0 , 8 6}$ & - & - & - & - \\
\hline Inga stenoptera Bentham 1381 & & 0,26 & 0,6 & 0,06 & $\mathbf{0 , 9 2}$ & 0,15 & 0,34 & 0,24 & $\mathbf{0 , 7 3}$ \\
\hline Inga tessmannii Harms 1551 & & 0,39 & 0,6 & 0,38 & 1,37 & 0,23 & 0,51 & 0,07 & $\mathbf{0 , 8 1}$ \\
\hline Inga vismiifolia Poeppig 5242 & $\mathrm{~L}$ & 1,11 & 1,2 & 0,44 & 2,76 & 0,98 & 1,53 & 1,85 & 4,35 \\
\hline Inga unidentified & & 0,07 & 0 & 0,4 & $\mathbf{0 , 4 7}$ & - & - & - & - \\
\hline $\begin{array}{l}\text { Zygia inaequalis (H. \& B. } \\
\text { Ex Willd.) Pittier } 8148\end{array}$ & & - & - & - & - & 0,23 & 0,34 & 0,48 & 1,05 \\
\hline $\begin{array}{l}\text { Zygia juruana (Harms) } \\
\text { L. Rico } 1467\end{array}$ & $\mathrm{~L}$ & 0,13 & 0,3 & 0,48 & 0,91 & 1,96 & 1,7 & 4,79 & 8,45 \\
\hline \multicolumn{10}{|l|}{ MORACEAE } \\
\hline $\begin{array}{l}\text { Brosimum guianense } \\
\text { (Aublet) Huber } 1333\end{array}$ & & 0,13 & 0,3 & 0,03 & 0,46 & 0,15 & 0,17 & 0,02 & 0,34 \\
\hline $\begin{array}{l}\text { Brosimum lactescens } \\
\text { S. Moore } 4097\end{array}$ & & 0,07 & 0,15 & 0,28 & 0,49 & - & - & - & - \\
\hline Clarisia biflora R. \& P. 1049 & & 0,26 & 0,45 & 0,22 & 0,93 & - & - & - & - \\
\hline
\end{tabular}

continúa... 


\begin{tabular}{|c|c|c|c|c|c|c|c|c|c|}
\hline Ficus maxima Miller N217296 & & 0,59 & 0,75 & 0,63 & 1,97 & 0,08 & 0,17 & 0,01 & 0,25 \\
\hline $\begin{array}{l}\text { Ficus paraensis } \\
\text { (Miquel) Miquel } 1364\end{array}$ & & 0,07 & 0,15 & 0,07 & 0,28 & - & - & - & - \\
\hline $\begin{array}{l}\text { Maclura tinctoria ssp. Tinctoria } \\
\text { (L,) Steudel N407489 }\end{array}$ & & 0,07 & 0,15 & 0,03 & 0,25 & - & - & - & - \\
\hline $\begin{array}{l}\text { Maquira coriacea } \\
\text { (Karsten) C.C. Berg } 2018\end{array}$ & $\mathrm{H}$ & 3,28 & 1,5 & 2,62 & 7,4 & 0,3 & 0,51 & 0,41 & 1,23 \\
\hline $\begin{array}{l}\text { Naucleopsis glabra } \\
\text { Spruce ex Pittier N307160 }\end{array}$ & $\mathrm{H}$ & 1,25 & 1,2 & 0,53 & 2,98 & 0,08 & 0,17 & 0,11 & 0,36 \\
\hline $\begin{array}{l}\text { Perebea longipedunculata } \\
\text { C.C. Berg } 1217\end{array}$ & $\mathrm{R}$ & 2,56 & 1,5 & 3,11 & 7,17 & 0,98 & 1,19 & 1,37 & 3,54 \\
\hline Sorocea steinbachii C.C. Berg 1141 & $\mathrm{H}$ & 2,62 & 1,5 & 3,7 & 7,82 & 0,75 & 1,36 & 0,8 & 2,91 \\
\hline Trophis racemosa (L.) Urban 2106 & & 0,26 & 0,6 & 0,65 & 1,51 & 0,23 & 0,34 & 0,9 & 1,46 \\
\hline \multicolumn{10}{|l|}{ MYRISTICACEAE } \\
\hline Iryanthera juruensis Warburg 7467 & $\mathrm{~L}$ & 0,26 & 0,45 & 0,13 & $\mathbf{0 , 8 4}$ & 2,41 & 1,02 & 3,58 & 7,01 \\
\hline $\begin{array}{l}\text { Virola elongata (Bentham) } \\
\text { Warburg } 6500\end{array}$ & & 0,2 & 0,3 & 0,47 & 0,97 & 0,23 & 0,51 & 0,26 & 1 \\
\hline $\begin{array}{l}\text { Virola pavonis (A. DC.) } \\
\text { A.C. Smith } 8454\end{array}$ & & 0,79 & 1,05 & 0,53 & 2,37 & 0,45 & 0,68 & 0,52 & 1,65 \\
\hline Virola unidentified & & 0,07 & 0 & 0,21 & 0,27 & - & - & - & - \\
\hline \multicolumn{10}{|l|}{ MYRSINACEAE } \\
\hline Stylogyne sp. 3463 & & 0,26 & 0,3 & 0,28 & $\mathbf{0 , 8 4}$ & 0,98 & 1,19 & 0,72 & 2,89 \\
\hline \multicolumn{10}{|l|}{ MYRTACEAE } \\
\hline Calyptranthes sp. 9388 & & 0,39 & 0,6 & 0,49 & 1,48 & 0,3 & 0,68 & 0,7 & 1,68 \\
\hline Calyptranthes sp. 2 N 219309 & & 0,13 & 0,3 & 0,46 & $\mathbf{0 , 8 9}$ & - & - & - & - \\
\hline Eugenia marowijensis Miquel 2351 & & 0,26 & 0,6 & 0,37 & 1,23 & 0,3 & 0,68 & 0,34 & 1,32 \\
\hline Eugenia muricata DC. 1091 & & 0,2 & 0,45 & 0,32 & 0,97 & 0,3 & 0,51 & 0,13 & 0,94 \\
\hline Eugenia ochrophloea Diels 4347 & $\mathrm{~L}$ & 0,66 & 0,9 & 0,71 & 2,26 & 2,03 & 1,7 & 2,53 & 6,27 \\
\hline Eugenia patens Poiret N 109216 & & 0,07 & 0,15 & 0,05 & $\mathbf{0 , 2 7}$ & 0,23 & 0,51 & 0,16 & 0,9 \\
\hline Eugenia sp. 21190 & & 0,07 & 0,15 & 0,01 & 0,22 & 0,15 & 0,34 & 0,42 & 0,91 \\
\hline Eugenia sp. 49045 & & 0,2 & 0,3 & 0,08 & $\mathbf{0 , 5 8}$ & 0,68 & 1,02 & 0,24 & 1,94 \\
\hline Eugenia sp. 65503 & & - & - & - & - & 0,23 & 0,51 & 0,01 & $\mathbf{0 , 7 5}$ \\
\hline Eugenia unidentified & & - & - & - & - & 0,08 & 0 & 0,42 & $\mathbf{0 , 5}$ \\
\hline Marliera subulata Mc Vaugh 7519 & & - & - & - & - & 0,08 & 0,17 & 0 & 0,25 \\
\hline Myrcia sp. 57347 & $\mathrm{~L}$ & - & - & - & - & 0,9 & 1,19 & 1,03 & 3,12 \\
\hline Myrcia sp. 78299 & & - & - & - & - & 0,53 & 0,51 & 0,67 & 1,7 \\
\hline $\begin{array}{l}\text { Myrciaria floribunda } \\
\text { (West ex Willdenow) O. Berg } 7045\end{array}$ & & - & - & - & - & 0,08 & 0,17 & 0,09 & 0,34 \\
\hline \multicolumn{10}{|l|}{ NYCTAGINACEAE } \\
\hline Neea floribunda Diels 8667 & $\mathrm{R}$ & 1,38 & 1,65 & 1,33 & 4,36 & 1,66 & 1,53 & 1,34 & 4,52 \\
\hline Neea sp. 9089 & & - & - & - & - & 0,23 & 0,34 & 0,59 & 1,16 \\
\hline \multicolumn{10}{|l|}{ OCHNACEAE } \\
\hline Ouratea sp. 5256 & & 0,13 & 0,3 & 0,02 & 0,45 & 0,3 & 0,51 & 0,11 & $\mathbf{0 , 9 2}$ \\
\hline
\end{tabular}


ESTRUCTURA Y COMPOSICIÓN FLORÍSTICA DEL BOSQUE DE LA LLANURA ALUVIAL

\begin{tabular}{|c|c|c|c|c|c|c|c|c|c|}
\hline OLACACEAE & & & & & & & & & \\
\hline $\begin{array}{l}\text { Cathedra acuminata (Bentham) } \\
\text { Miers } 7397\end{array}$ & & 0,07 & 0,15 & 0,03 & 0,24 & 0,08 & 0,17 & 0,34 & $\mathbf{0 , 5 9}$ \\
\hline $\begin{array}{l}\text { Heisteria acuminata (Humboldt \& } \\
\text { Bonpland) Engler N807283 }\end{array}$ & & 6,16 & 1,8 & 0,93 & 8,89 & 9,5 & 2,04 & 2,29 & 13,82 \\
\hline Heisteria spruceana Engler 5599 & & - & - & - & - & 0,45 & 0,51 & 0,06 & $\mathbf{1 , 0 3}$ \\
\hline Minquartia guianensis Aublet 2223 & & 0,39 & 0,75 & 0,69 & 1,84 & 0,08 & 0,17 & 0,09 & $\mathbf{0 , 3 3}$ \\
\hline PASSIFLORACEAE & & & & & & & & & \\
\hline Dilkea sp. & & 0,07 & 0,15 & 0,01 & $\mathbf{0 , 2 2}$ & - & - & - & - \\
\hline POLYGONACEAE & & & & & & & & & \\
\hline $\begin{array}{l}\text { Coccoloba densifrons C. Martius } \\
\text { ex Meissner } 5274\end{array}$ & & 0,72 & 1,05 & 0,73 & 2,5 & 1,66 & 1,19 & 1,83 & 4,68 \\
\hline Coccoloba lehmannii Lindau 9006 & & - & - & - & - & 0,08 & 0,17 & 0,12 & $\mathbf{0 , 3 6}$ \\
\hline Coccoloba mollis Casaretto 2019 & & 0,07 & 0,15 & 0,45 & 0,66 & 0,08 & 0,17 & 0,01 & 0,25 \\
\hline Coccoloba sp. 39042 & & 0,2 & 0,45 & 0,21 & $\mathbf{0 , 8 6}$ & 0,3 & 0,34 & 0,45 & 1,09 \\
\hline Triplaris americana L. 2122 & & 0,2 & 0,45 & 0,07 & $\mathbf{0 , 7 2}$ & - & - & - & - \\
\hline QUINACEAE & & & & & & & & & \\
\hline Quiina sp. 8398 & & 0,13 & 0,3 & 0,01 & $\mathbf{0 , 4 4}$ & 0,15 & 0,34 & 0,1 & $\mathbf{0 , 5 9}$ \\
\hline RUBIACEAE & & & & & & & & & \\
\hline $\begin{array}{l}\text { Alibertia edulis (Richard) } \\
\text { A. Richard ex DC. } 9166\end{array}$ & & 0,07 & 0,15 & 0,02 & 0,24 & 0,45 & 0,68 & 0,15 & 1,28 \\
\hline Alibertia sp. N407523 & & 0,2 & 0,3 & 0,04 & $\mathbf{0 , 5 4}$ & - & - & - & - \\
\hline Borojoa sp. 11552 & & - & - & - & - & 0,3 & 0,51 & 0,31 & 1,12 \\
\hline Chomelia barbellata Standley 1416 & & 0,13 & 0,3 & 0,21 & 0,64 & 0,23 & 0,51 & 0,21 & 0,95 \\
\hline Coussarea brevicaulis Krause 9385 & $\mathrm{R}$ & 1,64 & 1,65 & 1,2 & 4,49 & 2,79 & 1,36 & 2,12 & 6,27 \\
\hline Faramea sp. 1 & & - & - & - & - & 0,15 & 0,17 & 0,02 & $\mathbf{0 , 3 4}$ \\
\hline Faramea sp. 2 & & - & - & - & - & 0,15 & 0,17 & 0,03 & $\mathbf{0 , 3 5}$ \\
\hline Posoqueria sp. 1 N219479 & & 0,66 & 0,75 & 0,22 & 1,63 & 0,08 & 0,17 & 0,06 & $\mathbf{0 , 3 1}$ \\
\hline Posoqueria sp. 2 & & 0,07 & 0,15 & 0,03 & 0,25 & - & - & - & - \\
\hline Psychotria marginata Swartz 1246 & & 0,85 & 1,2 & 0,32 & 2,37 & 0,15 & 0,34 & 0,35 & 0,84 \\
\hline $\begin{array}{l}\text { Psychotria mathewsii } \\
\text { P. C. Standley }\end{array}$ & & - & - & - & $\mathbf{0}$ & 0,08 & 0,17 & 0,11 & $\mathbf{0 , 3 6}$ \\
\hline Psychotria remota Bentham 1365 & & 0,2 & 0,45 & 0,34 & 0,99 & 0,53 & 0,85 & 0,11 & 1,49 \\
\hline $\begin{array}{l}\text { Psychotria stenostachya } \\
\text { Standley N417200 }\end{array}$ & & 0,72 & 0,75 & 0,04 & 1,51 & - & - & - & - \\
\hline Randia armata (Swartz) DC. 8457 & & 0,33 & 0,6 & 0,12 & 1,05 & 0,15 & 0,34 & 0,36 & $\mathbf{0 , 8 5}$ \\
\hline Rondeletia sp. N309246 & $\mathrm{H}$ & 2,49 & 1,65 & 0,73 & 4,87 & 0,3 & 0,68 & 0,03 & 1,01 \\
\hline $\begin{array}{l}\text { Rudgea sessiliflora } \text { Standley } \\
\text { N821419 }\end{array}$ & $\mathrm{L}$ & - & - & - & $\mathbf{0}$ & 4,9 & 1,87 & 0,43 & 7,2 \\
\hline Simira sp. 9731 & & - & - & - & $\mathbf{0}$ & 0,15 & 0,34 & 0,07 & $\mathbf{0 , 5 6}$ \\
\hline SAPINDACEAE & & & & & & & & & \\
\hline Cupania latifolia H.B.K. 1507 & & - & - & - & $\mathbf{0}$ & 0,08 & 0,17 & 0,1 & $\mathbf{0 , 3 5}$ \\
\hline Talisia sp. 1 N317266 & & 0,92 & 1,05 & 0,8 & 2,77 & 0,53 & 0,85 & 0,53 & 1,91 \\
\hline
\end{tabular}




\begin{tabular}{|c|c|c|c|c|c|c|c|c|c|}
\hline Talisia sp. 2 N317267 & & 0,33 & 0,6 & 0,3 & 1,23 & 0,23 & 0,51 & 0,08 & $\mathbf{0 , 8 2}$ \\
\hline unidentified sp. 5548 & & - & - & - & $\mathbf{0}$ & 0,45 & 0,51 & 0,14 & 1,1 \\
\hline \multicolumn{10}{|l|}{ SAPOTACEAE } \\
\hline $\begin{array}{l}\text { Chrysophyllum argenteum ssp. } \\
\text { Auratum (Miquel) Pennington } 5295\end{array}$ & & 0,26 & 0,45 & 0,14 & $\mathbf{0 , 8 5}$ & 0,3 & 0,68 & 0,16 & 1,14 \\
\hline Chrysophyllum sp. 13282 & & 0,26 & 0,6 & 0,68 & 1,54 & - & - & - & - \\
\hline $\begin{array}{l}\text { Pouteria cuspidata ssp. Dura } \\
\text { (Eyma) Pennington } 5128\end{array}$ & & 0,2 & 0,3 & 0,12 & 0,62 & - & - & - & - \\
\hline $\begin{array}{l}\text { Pouteria reticulata } \\
\text { (Engler) Eyma } 2004\end{array}$ & & 0,66 & 1,2 & 0,49 & 2,35 & 1,21 & 1,36 & 2,21 & 4,78 \\
\hline Pouteria unidentified & & - & - & - & $\mathbf{0}$ & 0,15 & 0 & 0,4 & $\mathbf{0 , 5 5}$ \\
\hline $\begin{array}{l}\text { Sarcaulus brasiliensis ssp. } \\
\text { Brasiliensis (A. DC.) Eyma } 2336\end{array}$ & & 1,18 & 1,2 & 0,86 & 3,24 & 0,9 & 0,68 & 1,18 & 2,76 \\
\hline \multicolumn{10}{|l|}{ SIMAROUBACEAE } \\
\hline $\begin{array}{l}\text { Picramnia magnifolia } \\
\text { J.F. Macbr. N909191 }\end{array}$ & & - & - & - & - & 0,08 & 0,17 & 0,08 & $\mathbf{0 , 3 3}$ \\
\hline $\begin{array}{l}\text { Picramnia sellowii ssp. Spruceana } \\
\text { (Engler) Pirani N819372 }\end{array}$ & & 0,39 & 0,6 & 0,02 & 1,01 & 0,15 & 0,34 & 0,04 & $\mathbf{0 , 5 3}$ \\
\hline $\begin{array}{l}\text { Simaba orinocensis } \\
\text { H.B.K. N409099 }\end{array}$ & & 0,07 & 0,15 & 0 & 0,22 & - & - & - & - \\
\hline \multicolumn{10}{|l|}{ SOLANACEAE } \\
\hline Cyphomandra sp. & & 0,07 & 0,15 & 0,08 & $\mathbf{0 , 3}$ & - & - & - & - \\
\hline Solanum sp. 4 & $\mathrm{~L}$ & 0,79 & 0,75 & 0,14 & 1,68 & 3,17 & 1,87 & 0,74 & 5,78 \\
\hline Solanum sp. 8 & & 0,2 & 0,45 & 0,02 & 0,67 & 0,08 & 0,17 & 0 & 0,25 \\
\hline \multicolumn{10}{|l|}{ STERCULIACEAE } \\
\hline Herrania sp. & & 0,2 & 0,45 & 0,04 & 0,69 & 0,15 & 0,34 & 0,07 & 0,56 \\
\hline Sterculia sp. 3492 & & 0,33 & 0,15 & 0,05 & $\mathbf{0 , 5 3}$ & - & - & - & - \\
\hline Theobroma cacao L. 2016 & & 0,2 & 0,45 & 0,8 & 1,45 & - & - & - & - \\
\hline \multicolumn{10}{|l|}{ THEOPHRASTACEAE } \\
\hline Clavija sp. N219467 & & 0,33 & 0,6 & 0,04 & 0,97 & - & - & - & - \\
\hline \multicolumn{10}{|l|}{ TILIACEAE } \\
\hline $\begin{array}{l}\text { Luehea cymulosa Spruce } \\
\text { ex Bentham } 7084\end{array}$ & & - & - & - & - & 0,53 & 0,51 & 1,1 & 2,14 \\
\hline Vasivaea sp. 6556 & & - & - & - & - & 0,08 & 0,17 & 0,12 & $\mathbf{0 , 3 7}$ \\
\hline \multicolumn{10}{|l|}{ VIOLACEAE } \\
\hline $\begin{array}{l}\text { Gloeospermun equatoriense } \\
\text { Hekking } 2168\end{array}$ & $\mathrm{H}$ & 4,06 & 1,8 & 5,5 & 11,36 & 0,45 & 0,68 & 1,3 & 2,43 \\
\hline $\begin{array}{l}\text { Leonia glycycarpa Ruiz Lupez \& } \\
\text { Pavun } 2027\end{array}$ & $\mathrm{H}$ & 4,78 & 1,8 & 10,1 & 16,68 & 0,53 & 0,68 & 0,67 & 1,88 \\
\hline \multicolumn{10}{|l|}{ NO IDENTIFICADO } \\
\hline No identificado & & 0,72 & 0 & 0,54 & 1,26 & 2,11 & 0 & 1,87 & 3,98 \\
\hline Valores absolutos totales & & 1526 & 667 & 1,69 & & 1327 & 589 & 1,53 & \\
\hline
\end{tabular}


APÉNDICE 2. Clasificación de especies de acuerdo al máximo DAP alcanzado (MaxDAP) en el bosque de restinga. Se consideran solamente las especies presentes con más de 10 individuos. Las especies dentro de los grupos están ordenados alfabéticamente.

MaxDAP $\leq 10 \mathrm{~cm}$

Bactris sp.

Coussarea brevicaulis

Garcinia madrunio

Heisteria acuminata

Naucleopsis glabra

Picramnia sellowii ssp. Spruceana

Posoqueria sp. 1

Psychotria stenostachya

Rondeletia sp.

Rudgea sessiliflora

Solanum sp. 4

Tabernaemontana markgrafiana

Talisia sp. 1

Tococa coronata

Tococa sp.

$10 \mathrm{~cm}<\operatorname{MaxDAP} \leq 25 \mathrm{~cm}$

Anaxagorea sp.

Astrocaryum murimuri

Borojoa sp.

Byrsonima densa

Capparis sola

Casearia aculeata

Casearia sylvestris

Cecropia ficifolia

Chomelia barbellata

Chrysophyllum argenteum ssp. Auratum

Coccoloba densifrons

Cordia nodosa

Couroutari oligantha

Crematosperma sp.

Diospyros sp. 1
Eugenia marowijensis

Eugenia muricata

Eugenia sp. 4

Euterpe precatoria

Ficus maxima

Gloeospermum equatoriense

Hirtella triandra ssp. Triandra

Inga cinnamomea

Inga tessmannii

Iryanthera juruensis

Laetia corymbulosa

Leonia glycycarpa

Malmea sp.

Matisia bracteolosa

Myrcia sp. 5

Neea floribunda

Ocotea javitensis

Parinari parilis

Perebea longipedunculata

Pleurothyrium parviflorum

Pseudoxandra polyphleba

Psychotria marginata

Psychotria remota

Pterocarpus amazonum

Pterocarpus sp. 1

Randia armata

Rollinia cuspidata

Socratea exorhiza

Sorocea steinbachii

Stylogyne sp.

Swartzia cardiosperma

Swartzia simplex

Theobroma cacao

Trichilia rubra 
Triplaris amaricana

Trophis racemosa

Zygia juruana

$25 \mathrm{~cm}<\operatorname{MaxDAP} \leq 125 \mathrm{~cm}$

Alchornea schomburgkii

Andira inermis

Aniba sp. 1

Astrocaryum jauari

Canatola venezuelana

Calyptranthes sp. 1

Casearia arborea

Cathedra acuminata

Cecropia membranacea

Cedrela odorata

Clarisia biflora

Cordia lutea

Croton cuneatus

Cupania latifolia

Drypetes amazonica var. Peruviana

Duguetia spixiana

Eschweilera parvifolia

Eschweilera turbinata

Eugenia ochrophloea

Ficus killipii

Garcinia macrophylla

Guatteria sp. 1

Himatanthus bracteatus

Inga edulis

Inga nobilis

Inga semialata

Inga stenoptera

Inga vismiifolia

Luehea cymulosa

Maytenus macrocarpa
Minquartia guianensis

Mouriri grandiflora

Nectandra cuneato - cordata

Ocotea cernua

Oxandra sphaerocarpa

Platymiscium stipulare

Pourouma acuminata

Pourouma cecropiifolia

Pouteria reticulata

Protium nodulosum

Sapium glandulosum

Sapium marmierii

Sarcaulus brasiliensis ssp. Brasiliensis

Scheelea cephatotis

Sloanea guianensis

Spondias mombin sen. Lat.

Sterculia sp.

Trichilia pleeana

Unonopsis floribunda

Virola elongata

Virola pavonis

Xylopia micans

Xylopia sp. 1

Zygia cauliflora

$75 \mathrm{~cm}$ MaxDAP $\leq 125 \mathrm{~cm}$

Brosimum lactescens

Calycophyllum spruceanum

Ceiba samauma

Coccoloba sp. 3

Guarea macrophylla

Hura crepitans

Licania britteniana

Maquira coriacea

Terminalia oblonga 


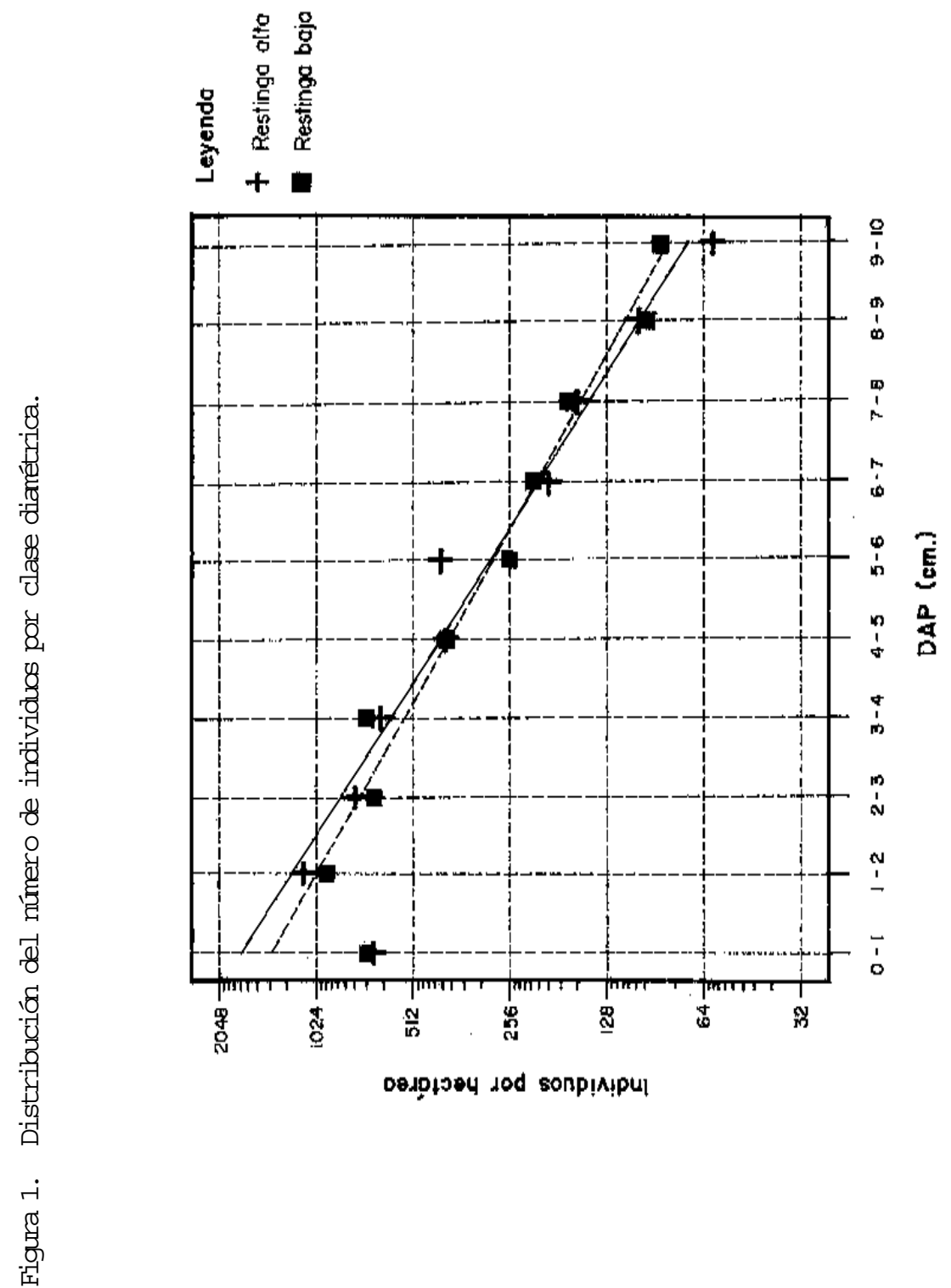




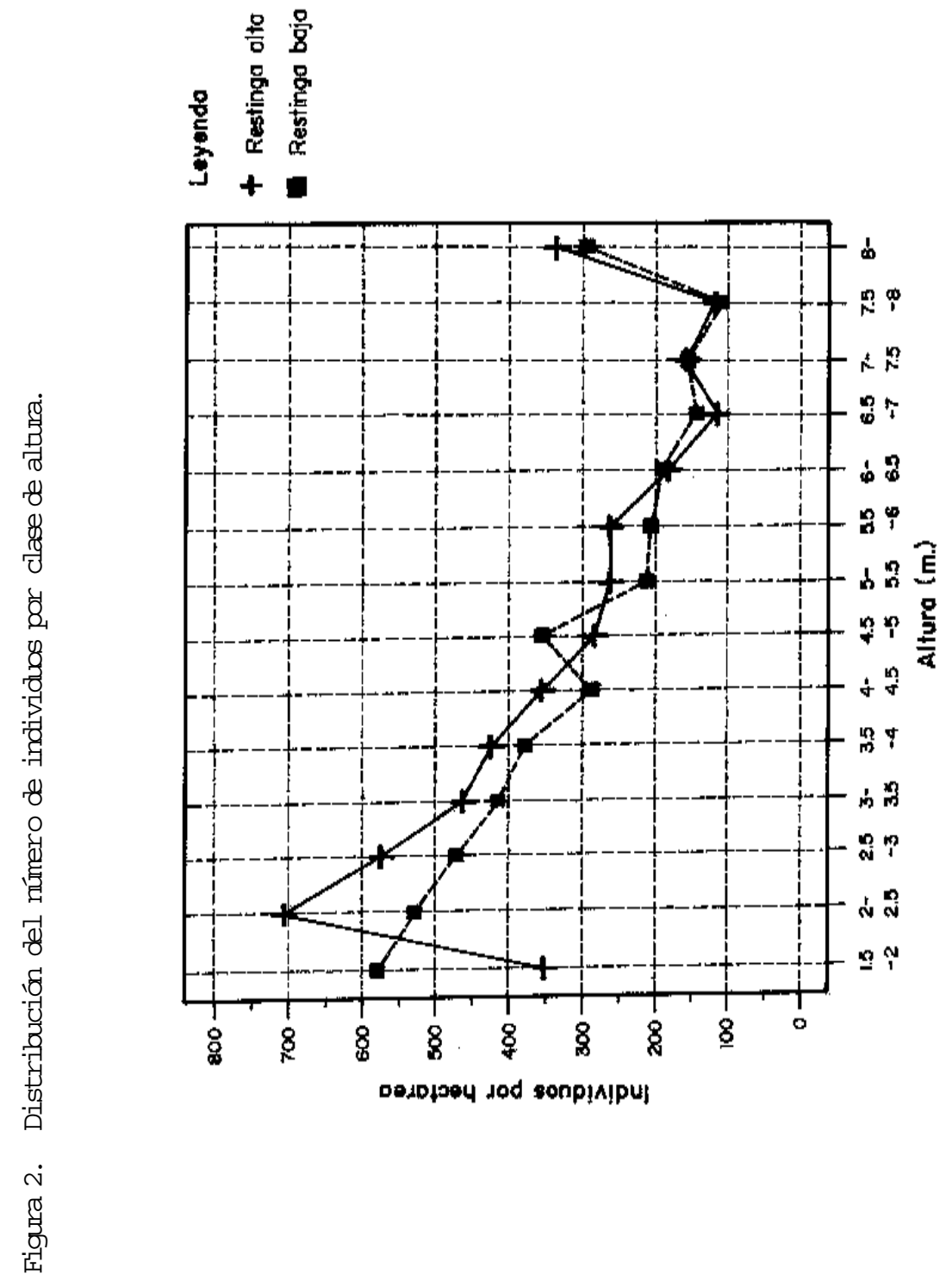




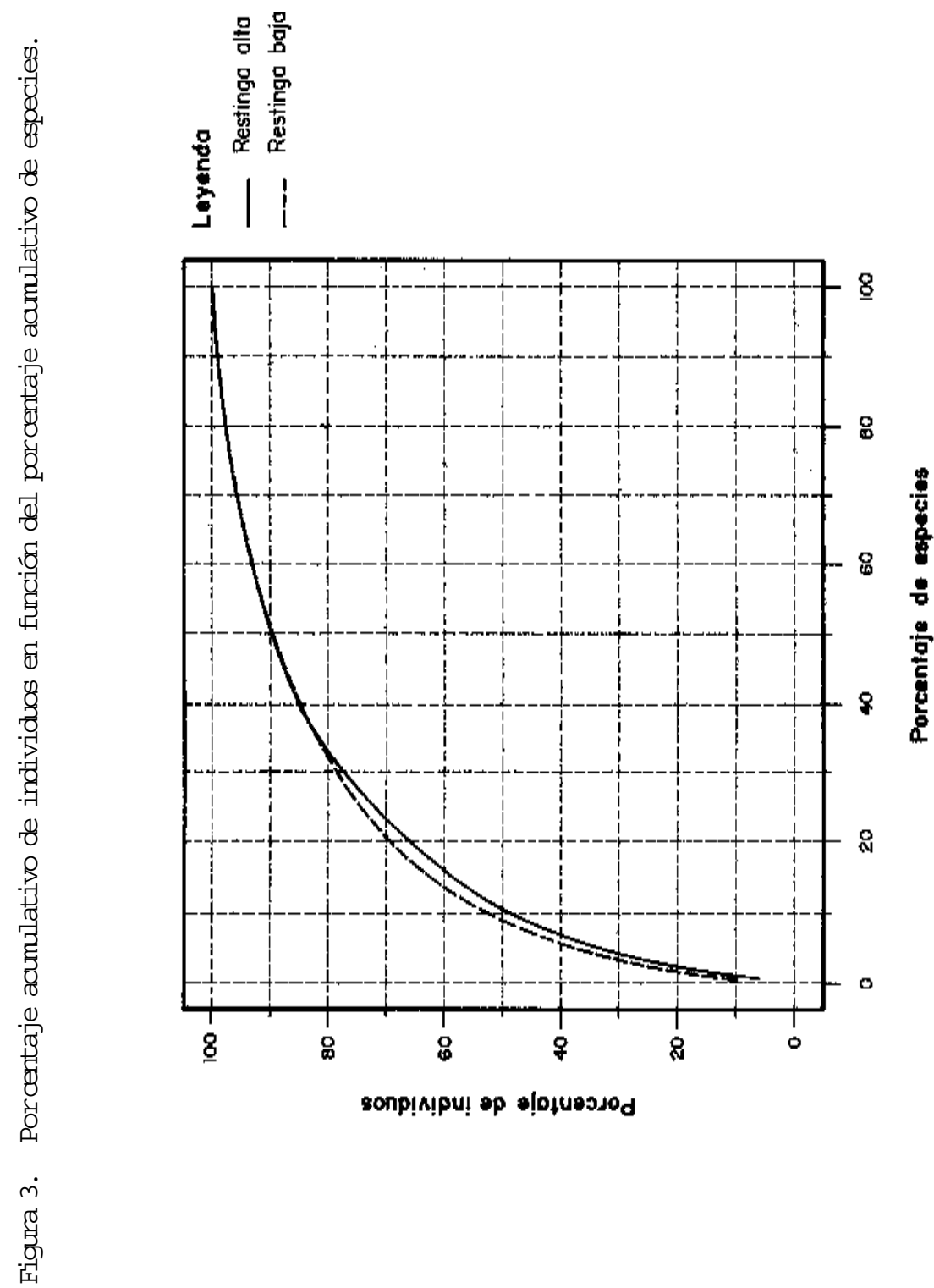




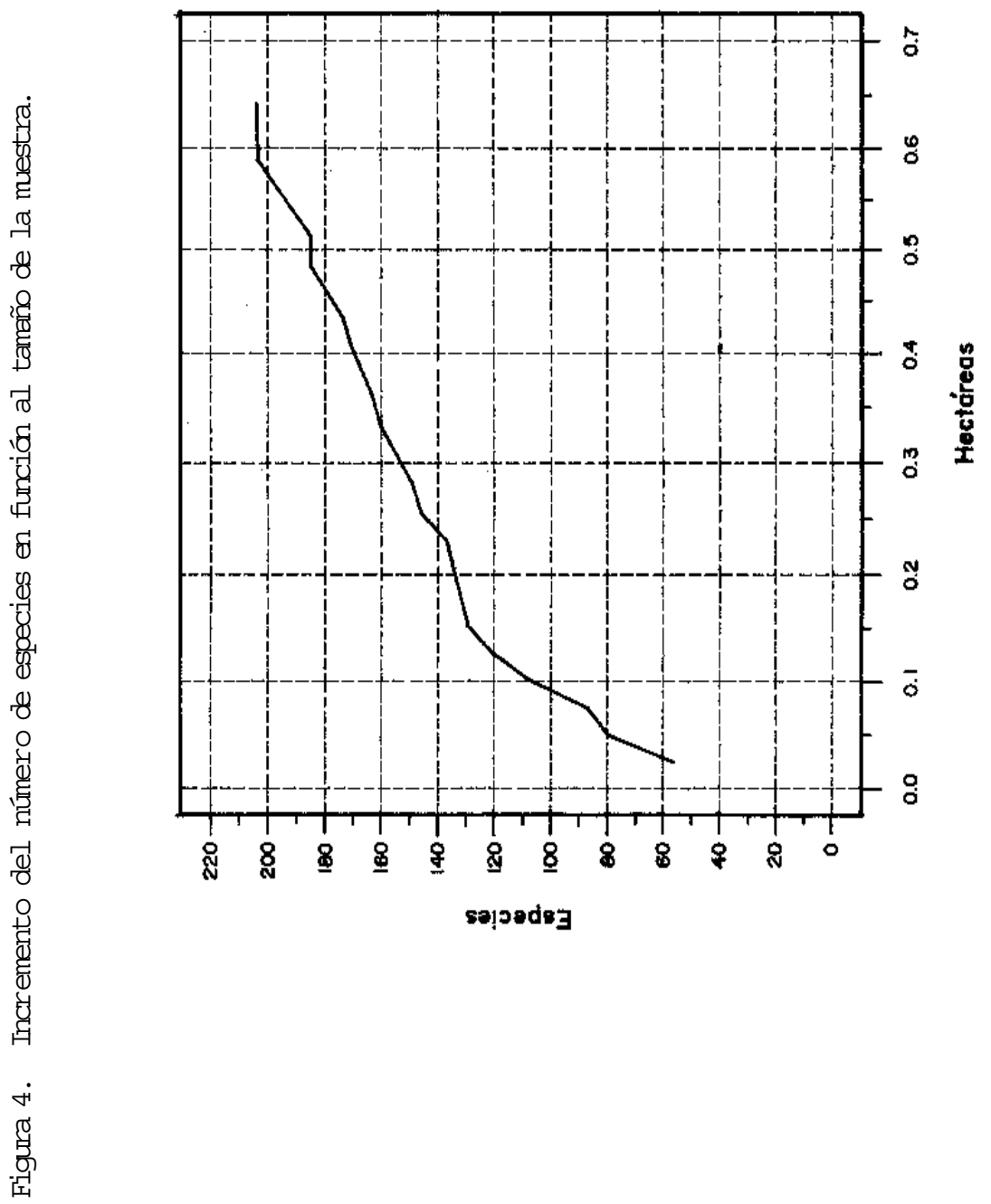




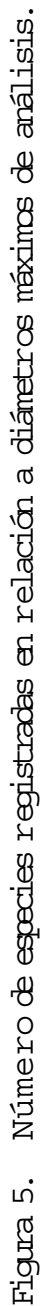

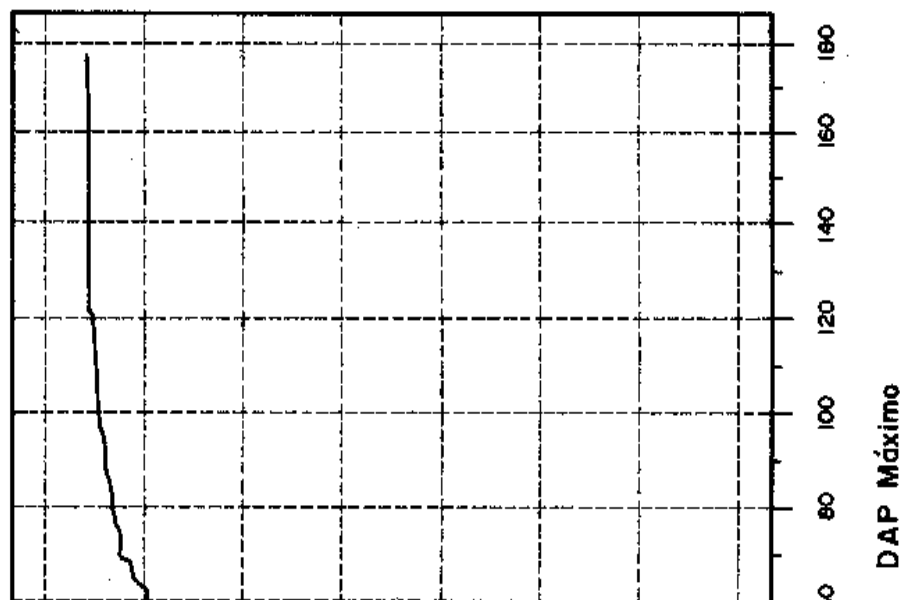

